\title{
The general causal cumulative model of ordinal response
}

\author{
Borysław Paulewicz • Agata Blaut
}

Received: date / Accepted: date

\begin{abstract}
In this paper, we introduce the general causal cumulative model of ordinal response. The statistical part of this model is a new family of hierarchical or non-hierarchical generalized linear models that represent the distribution of the outcome as a thresholded latent distribution. Its defining feature is the new link functions, which are order-preserving in the sense that they allow for arbitrary effects in individual thresholds while preserving their order. We show how the model can be interpreted as a generalization of some Signal Detection Theory (SDT) models and some Item Response Theory models. We propose an approach to measurement of latent variables which seems to follow from the requirement that measurements should be interpreted in the context of a causal theory of the unobservable response process. In particular, we formulate a causal definition of measurement invariance that seems to match the examples of measurement bias found in the literature, and we show that the commonly accepted statistical definition is flawed; this leads to the recognition of the fundamental problem associated with item parameters and of the critical role of substantive theory and speculative reasoning. We illustrate how, by making use of the statistical properties of our model, this approach to measurement can be followed in practice, and we explain what kind of issues of a more technical nature it may help address and when. In particular, we identify the central causal assumption of SDT models, we show how this assumption can be tested, how introducing item parameters can lead to severely biased point and interval estimates of the target causal quantities, and how causal estimate bias can be accounted for by means of a causal sensitivity analysis.
\end{abstract}

Keywords ordinal scale $\cdot$ measurement invariance $\cdot$ causal inference $\cdot$ alternative causal explanations $\cdot$ signal detection theory $\cdot$ item response theory $\cdot$ generalized linear models · hierarchical models · Bayesian inference

B. Paulewicz

Institute of Psychology, Jagiellonian University, Kraków, Poland

E-mail: boryslaw.paulewicz@uj.edu.pl

A. Blaut

Institute of Psychology, Jagiellonian University, Kraków, Poland 


\section{Introduction}

When a person answers a question by marking their response on a Likert-type scale, or when a decision is rated on confidence, or when a word in a recognition task is classified as previously presented or not ${ }^{1}$, or in a great number of other similar situations, the response is ordinal. This kind of response, which is extremely common in psychology, poses two closely related problems.

Firstly, there is a problem of scale: distances between points in the underlying space may not be preserved by the observed response categories when they are interpreted as numbers. Secondly, there is the often-neglected problem of interand intra-individual differences in the systematic part of the mapping between the latent variables and the responses; for instance, the same individual may, but only in some situations, exaggerate how pleasant, or unpleasant, certain experiences are by systematically using more extreme ratings. This is an example of what we call a difference in the way of responding.

The purpose of this paper is to introduce a new family of generalized linear models that can be used as part of various methods of analysis of ordinal binary or polytomous (i.e., with more than two possible values) response data in a wide range of settings. We believe that this model may be useful not only because it includes some important models as special cases, but primarily because, when it is supplemented with a causal interpretation, it can sometimes help address both of the aforementioned problems in a way that may be worthy of consideration.

We have tried to make parts of this paper accessible to a wider audience, which is why it is quite long. To this end, we introduce the relevant elements of Signal Detection Theory (Peterson, Birdsall, \& Fox, 1954; Tanner Jr \& Swets, 1954; Green \& Swets, 1966) and Item Response Theory (IRT, see Van der Linden, 2018, for a comprehensive introduction) almost from scratch. We have also tried to make the more technically involved parts as isolated as we could, which is why the orderpreserving link functions are defined in the Appendix. However, the target user of our model is a researcher with some knowledge of Bayesian inference, SDT or IRT, the generalized linear modelling framework, hierarchical models, and elementary causal inference (Pearl, 2000; Pearl, Glymour, \& Jewell, 2016).

For now, it is important to know that SDT models are simple models of the decision-making process, and that they are, in principle, applicable to data from arbitrary tasks that require classification; IRT is a part of Modern Test Theory (Junker \& Ellis, 1997), which is a comprehensive contemporary theory of testing as well as a family of measurement models. Loosely speaking, in SDT the main focus is on the decision process that leads to the response; in IRT, the focus is more on the instrument and its applications.

We should note at the outset that there have been some recent attempts at combining IRT models with dynamic generalizations of SDT. Most notably in our view, Tuerlinckx and Boeck (2005) showed that certain IRT models arise from the process described by the diffusion model (Ratcliff, 1978; Ratcliff \& McKoon, 2008) and van der Maas, Molenaar, Maris, Kievit, and Borsboom (2011) showed how this fact can be exploited in practice. We are also aware of two other attempts at combining causal inference with psychometrics, i.e., Causal Theory of Error Scores (CTES, van Bork, Rhemtulla, Sijtsma, \& Borsboom, 2020) and Situational

\footnotetext{
1 We will explain how a binary response can be ordinal later.
} 
Optimization Analysis framework (SOFA, Franco, Wiberg, \& Laros, 2021). CTES is a causal-theoretic account of various types of errors in tests composed of multiple items. In SOFA, which is an approach to psychological measurement, many of the same ingredients that we focus on here are combined. Like us, the authors of SOFA also emphasize the individual nature of the response process, they discuss some of the limitations of IRT in that regard, they incorporate the causal-theoretic perspective, and they provide a Bayesian implementation of their model.

The structure of the paper

We begin by introducing the cumulative family of models interpreted as a simplified representation of the process of categorisation of a latent variable. We insist that this interpretation was taken seriously, and we derive some consequences of this point of view. In particular, we analyse a range of situations in which this kind of model can be used, and we show how those can be viewed from a unified causal-theoretic perspective. This leads to the recognition of the implications of an important property of IRT models, i.e., of the presence of item parameters. We argue that this property makes it difficult, if not impossible, to properly address the issue of measurement invariance; we propose a causal definition of measurement invariance, we then conclude that the property of having item parameters may be the main reason that makes it impossible to interpret IRT models as simplified causal models of the response process, which leads us to a new definition of item effect. In the remaining part of the paper, we provide a detailed description of two causal analyses that serve to illustrate how combining the two parts of our model, i.e., the causal part and the statistical part, makes it possible to establish the validity or lack thereof of a substantive interpretation of the results.

\section{Ordinal response as a discrete order-preserving manifestation of points in a latent space}

In psychology studies, it is often the case that some latent property or state, such as the current mood, an attitude towards something, the perceived clarity of the stimulus, or confidence in the correctness of the decision, is expressed using ratings. To a first approximation, it can be assumed that the ratings correspond to latent psychological attributes or states that can be thought of as points in a continuous linearly ordered metric space.

For this kind of measurement to have the intended meaning, the relation between the latent space and the space of ratings has to be approximately orderpreserving, meaning that if $l_{1}<l_{2}$ then $p\left(R \geq r \mid l_{1}\right) \leq p\left(R \geq r \mid l_{2}\right)$ for all $l_{1}, l_{2}$, and $r$, where $l_{i}$ is a value of the latent variable and $R$ is the response. In IRT, this assumption is called monotonicity; here, we rely on a much weaker assumption that we call local monotonicity. Local monotonicity holds when monotonicity holds for a particular individual in a particular situation; it does not require that monotonicity holds if $l_{1}$ and $l_{2}$ are values of the latent variable of different groups of individuals, or of different individuals, or of the same individual in different situations. We do not mean to suggest that local monotonicity always holds, or even that it sometimes holds; we only suggest that it may be a reasonable simplification 
because when ratings are observed, even a relatively weak interpretation of what they may indicate often requires that this assumption is at least approximately true.

Since the ratings are discrete, the response process is a special case of classification of internal states. For example, the space of all the possible internal feelings of excitement can be thought as a continuum; a particular individual in a particular moment in time may classify the internal feeling of excitement at that time as "moderate" or "intense". Since we assume that the latent space is linearly ordered, it is natural to assume that there exist ordered latent decision thresholds that determine the mapping between the latent space and the response space.

This is a causal model in so much as it is thought to describe, in a simplified way, the psychological response process. Note, however, that this kind of realist way of thinking about the family of models that we are gradually introducing is always present, e.g., in SDT, but it does not seem to be commonly entertained, at least not explicitly, in IRT. The reasons why it is not will become clear later.

According to this model, rating $k$ is given when an internal state, which is formally a point in the latent space, falls between the thresholds, which are formally points in the same space, and which define the interval corresponding to $k$. For instance, I (meaning the first author) may respond with "almost never" (encoded as 1) to the item "I wish I could be as happy as others seem to be" from the STAI questionnaire (Spielberger, Gorusch, Lushene, Vagg, \& Jacobs, 1983), because, when I try to think about this item, all that comes to my mind is a vague sense of indifference (a point in the latent space or an internal experience sample) that to me (I have my own set of thresholds) seems to be more accurately expressed by the lowest possible rating "almost never" than by "sometimes" (encoded as 2), which is one step higher.

As illustrated in Fig. 1 below, the part of the model introduced so far already formally captures the limitation of ordinal scale: when ratings are expressed or encoded using numbers, there is a well-defined notion of distance between ratings as numbers, but the distances between the ratings may not preserve the corresponding distances between points in the latent space.

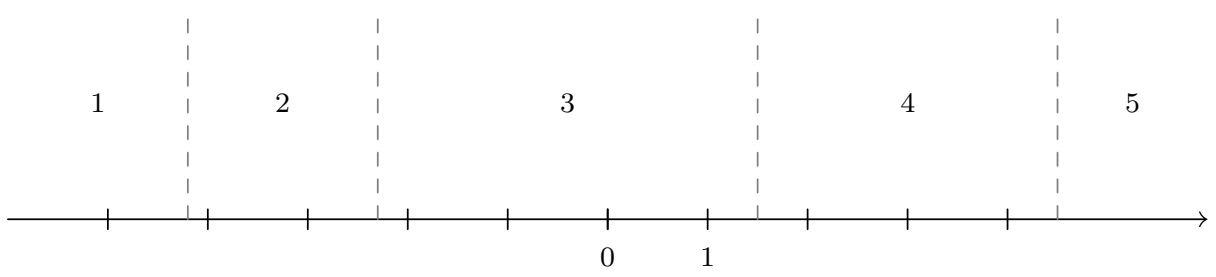

Latent scale

Fig. 1: Ordinal scale as a discretized continuous scale. The numbers above the axis are rating categories.

Here, the difference between ratings 1 and 2 interpreted as numbers is the same as the difference between ratings 2 and 3 interpreted as numbers, but the two pairs of ratings may arise from pairs of points in the latent space that are not equidistant. 
That the distances in the latent space may not be preserved by the responses has nothing to do with the fact that the response space is discrete; the problem is one of nonlinearity, not of discreteness. If the response was continuous and the relation between the latent variable $L$ and the response $R$ was given by $R=L^{3}$, the same problem would arise. The scale is ordinal also regardless of the labels used to denote the response categories, as long as there is some relevant notion of order of the response categories in sight. For instance, from the perspective of an SDT model, calling the two kinds of response to a binary classification task "noise" and "signal" instead of "1" and "2" changes nothing. In particular, it does not make the response nominal; it merely hides the fact that one response category corresponds to higher internal evidence of observing some "signal", which is as good a notion of order as any other.

The latent distributions

Even when the latent dimension is defined in the sense that it has an origin and a unit, both of which can be arbitrary, the model does not yet correspond to any response distribution because the probability of response $k$ is undefined unless the thresholds are defined, and the probability of the latent point falling between the corresponding thresholds is also defined. This probability is defined, and the idea is turned into a testable model once a latent distribution is introduced. In Fig. 2 below, there are two such models.
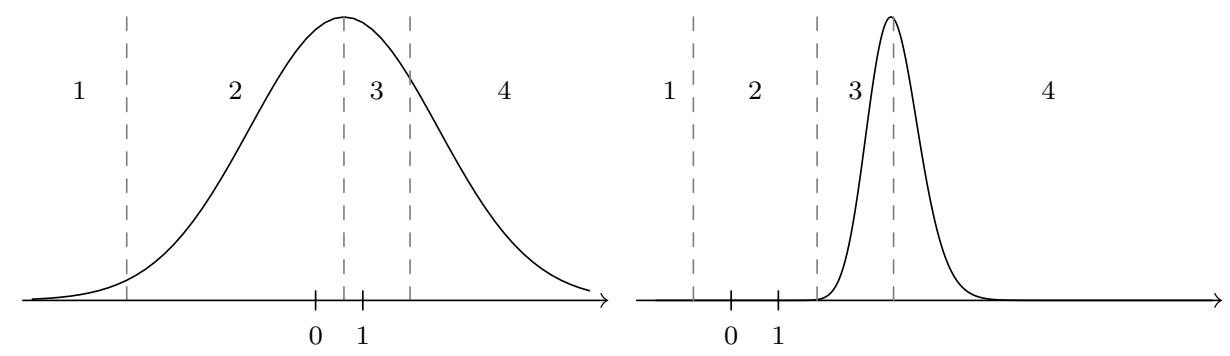

Fig. 2: Two cumulative models. The horizontal axis represents the underlying latent space, the dashed lines are the thresholds, the numbers above the axis are rating categories, and the density curves are the distributions of latent points.

The two models in Fig. 2 are members of the cumulative family (Mellenbergh, 1995; Van Der Ark, 2001), called this way because the probability of response $k$ can be expressed in terms of a function that gives the area under the density curve to the left of the thresholds, i.e., in terms of a cumulative distribution function $(\mathrm{CDF})$ :

$$
p(k \mid \tau, \theta)=F\left(\tau_{k} \mid \theta\right)-F\left(\tau_{k-1} \mid \theta\right)
$$

where $\boldsymbol{\tau}_{i}$ is the $i$-th threshold, $\boldsymbol{\tau}_{i}>\boldsymbol{\tau}_{j}$ if $i>j, \boldsymbol{\tau}_{0}=-\infty, \boldsymbol{\tau}_{K}=\infty, K$ is the number of response categories, and $F$ is the CDF of the latent variable. Here, 
the CFD is parametrized by a scalar $(\theta)$ but in general it may be a vector. For example, the probability of response 2 in the first model in Fig. 2, which is given by the area under the density curve between the first and the second threshold, can be obtained by subtracting the area under the density curve to the left of the first threshold from the area under the density curve to the left from the second threshold.

All SDT models are generalizations of some cumulative model; it is also one of the two most common types of model in IRT; the other is the adjacent category model (Mellenbergh, 1995; Van Der Ark, 2001). For instance, when there is one threshold, and the latent distribution is the standard normal distribution it is the Normal Ogive Rasch model (Rasch, 1960) also known as the 1PL model; when there are multiple ordered thresholds and the latent distribution is the standard logistic distribution it is the Graded Response Model (Samejima, 1968).

Although a generic cumulative IRT model can be interpreted as a formalized part of a general theory of measurement, sort of like a "blueprint for a way of thinking about psychological measurement" (Borsboom, 2005), when a method that is supposed to be described by an IRT model is used, the model has to be interpreted as a candidate causal model of the response process. Consider, for example, an experimental study in which a randomly assigned variable $X$ is hypothesized to influence a latent variable $A$, which is hypothesized to influence some other latent variable $B$, which is supposed to be measured by $Y$. If this is a psychological experiment, the researcher will most likely have to seriously consider the possibility that there are some other hard to identify latent variables or processes that may create additional causal paths from $X$ to $Y$, i.e., $X \rightarrow A \rightarrow$ $Y, X \rightarrow B \rightarrow Y$, or $X \rightarrow Y$. We will provide a realistic example of exactly this kind of situation in the context of the first causal analysis, but now we only note that the fact that typically both $X \rightarrow A \rightarrow Y$ and $X \rightarrow Y$ have to be considered, and it may not be clear what processes correspond to either path, means that the researcher cannot rely on the known properties of any measurement model that aims to describe $B \rightarrow Y$.

The reason that this is usually the case is that in psychology, we do not know enough about the measurement process to safely assume that measurement error is just random noise in the sense that it is not related to anything else that may be relevant in the given context. For instance, a person might respond in some way to the items on a personality or on an ability test for many reasons other than the personality trait or the ability that is supposed to be measured by the test, but most if not all such reasons (e.g., learning, fatigue, specific interests, specific skills, motivation, boredom, the attitude towards the person who administers the test, personal meaning attached to the items, etc.) are anything but random. In this last example, the intent was to find evidence of the existence of the $A \rightarrow B$ arrow by estimating the causal effect due to the $X \rightarrow A \rightarrow B \rightarrow Y$ path, but if no part of the causal model is known well enough, this intent is the only reason to think about $B \rightarrow Y$ as a measurement (sub)model. Needless to say, the validity of a substantive interpretation of estimates does not depend on what anyone is aiming to achieve.

The latent distribution in a cumulative model can be interpreted causally in several different ways (see, e.g., Holland, 1990; van Bork et al., 2020, for a similar perspective). Firstly, it can be thought of as the distribution of internal states of a single individual across situations. This will be the common choice when, e.g., 
repeatedly presented stimuli are rated on some discrete scale. Secondly, it can be thought of as the distribution of approximately constant attributes of different individuals. This will be the common choice when, e.g., the ratings are responses on a self-report measure of some trait. Finally, it can be thought of as the distribution of internal samples of state such that the properties of this distribution come from a distribution of internal meta-states, e.g., trait-like attributes, of the same individual. This hierarchical view seems like the natural choice when, e.g., the ratings are multiple responses on a self-report measure of current mood, collected during an extended period of time, during which different events may occur that may significantly affect the mood.

We suggest that the latent distribution should be primarily thought of as a context-dependent distribution of internal samples of state of a particular individual. There are at least two reasons for that: A latent trait-like variable cannot just manifest itself directly through the response to an item or a stimulus. For instance, before a relatively stable state of having a particular level of intelligence manifests itself through the response, a series of more transient intermediate states has to occur, and none of those states is intelligence. In particular, the response category has to be chosen on the basis of some internal source of information, such as the perceived stimulus, a recollection of some state of affairs in the past, or the result of some thought process. This internal source, which is anything but constant, is set up by the individual as means to provide the response in a way that, according to this individual, is appropriate in the given context. Hence the term "internal sampling". Moreover, any trait-like variable has to be ultimately defined in terms of behaviour, but, in general, internal states or types of behaviour do not have to be defined in terms of traits. Mood may be a good example of this: it may change quickly or slowly in the same time interval but there seems to sometimes be a need to use the notion of mood as the typical value of current mood in some extended period of time, as well as a need to sometimes use the notion of mood as something perhaps even more stable, e.g., as the proclivity to feel happy or sad. All the aforementioned mood-related notions are defined in terms of current mood, however.

Since we propose to interpret our model as a simplified representation of the response process, we also have to introduce the latent distribution of thresholds. Essentially, the same range of viewpoints about the sources of variability can be considered in this case, leading to the same general conclusion. That is because, as long as the model is causal, the thresholds are also a part of the internal response process. In particular, it is realistic to assume that the same individual can use the same rating categories differently in different situations, in the sense of there being some context-dependend differences in the systematic part of the mapping between the space of internal samples and the space of response categories.

In the next section, we provide the formal definition of a new family of cumulative regression models, which constitute the statistical part of the general causal cumulative model of ordinal response.

\section{The formal definition of the general cumulative regression model}

As long as the thresholds are fixed, modelling the effects in the latent variable usually does not pose any special difficulties. However, to model the effects in ordered 
thresholds using a hierarchical or a non-hierarchical linear regression structure, the thresholds have to be represented by unconstrained parameters because the coefficients of a linear regression model are in general not constrained. Otherwise, the results of naively attaching a linear regression structure will make sense only in special cases, if at all.

To see why, assume that the thresholds were naively treated as unconstrained parameters. If each threshold was modelled as related to a numerical predictor in a way that can be represented by arbitrary linear regression slopes, then if the slopes were not exactly the same, the model would imply that above and below some value of the predictor the order of the thresholds is reversed. If each threshold was modelled as related to a nominal or a numerical predictor in a way that can be represented by arbitrary linear regression coefficients, then interval estimates would not be forced to respect the constraints implied by the ordering. This is bound to lead to inference that is sometimes nonsensical, just like fitting a linear model to binary data is bound to sometimes produce interval estimates that extend outside the lower (0) or the upper (1) boundary. If the linear model was further extended by adding a hierarchical regression structure, a similar problem would occur. That is because in hierarchical linear regression, random effects are assumed to be normally distributed, and normal distribution has unbounded support. Naively representing random effects in multiple ordered thresholds as normally distributed would imply that every threshold can switch places with every other threshold.

The statistical part of the general causal cumulative model describes the distribution of response in terms of an arbitrary latent distribution and a vector of ordered thresholds. An isomorphic link function $f$ maps the space of vectors of thresholds $\boldsymbol{\tau}$ of dimension $K-1$ such that $\boldsymbol{\tau}_{i}<\boldsymbol{\tau}_{j}$ if $i<j$, where $K$ is the number of response categories, to an unconstrained space of vectors $\gamma$ of the same dimension.

The parameters that define the latent distribution can be fixed, free, or regressed, perhaps via suitable link functions (as in, e.g., Paulewicz \& Blaut, 2020) on arbitrary predictors. We do not present this regression structure as part of the formal definition here because it is essentially the same as the part that we define, except that the link functions are arbitrary, and when the latent distribution is defined by a parameter vector there may be more than one regression structure, and each regression structure can represent the effects in different elements of the parameter vector. For instance, the Unequal Variance (UV) Normal SDT model can be obtained as a special case if the latent distribution is normal and at least three separate regression structures are defined: one for the effects in the thresholds, one for the effects in $d^{\prime}$ (typically in the log space, see Paulewicz \& Blaut, 2020), and one for the effects in, e.g., the logarithm of the ratio of the standard deviations of the two distributions of internal evidence samples. Below, we define the hierarchical version of the model, but the hierarchical part can of course be omitted. 


$$
\begin{aligned}
\boldsymbol{\gamma}_{i, \cdot} & =\boldsymbol{X}_{i, \cdot} \boldsymbol{\beta}+\boldsymbol{Z}_{i, .} \boldsymbol{\delta} \\
f^{-1}\left(\boldsymbol{\gamma}_{i, .}\right) & =\boldsymbol{\tau}_{i, \cdot} \\
p\left(\boldsymbol{y}_{i}=k\right) & =F\left(\boldsymbol{\tau}_{i, k} \mid \boldsymbol{\theta}\right)-F\left(\boldsymbol{\tau}_{i, k-1} \mid \boldsymbol{\theta}\right)
\end{aligned}
$$

Here, $i=1 \ldots N$ is the observation number, $\boldsymbol{X}$ is the fixed effects model matrix, $\boldsymbol{Z}$ is the random effects model matrix, $\boldsymbol{\beta}$ and $\boldsymbol{\delta}$ are the fixed and random effects respectively, $\boldsymbol{\tau}$ is an $N \times K-1$ matrix, $\boldsymbol{y}$ is the response, $F$ is the cumulative distribution function of the latent variable parametrized by $\boldsymbol{\theta}$, and $f$ is the orderpreserving link function. Note that $\boldsymbol{\gamma}_{i}$, is in general a vector, and so $\boldsymbol{\beta}$ and $\boldsymbol{\delta}$ are matrices: The $j$-th rows of the $\boldsymbol{\beta}$ and $\boldsymbol{\delta}$ matrices represent fixed and random effects in the $j$-th element of the $\gamma$ vector.

The new link functions are described in the Appendix; the remaining details, including the softmax link function, the covariance of random effects, the priors, and the known limitations of the current implementation, are described in Paulewicz and Blaut (2020) and in the bhsdtr2 documentation.

\section{Scope of applicability of the general causal cumulative model}

In this paper, we consider four kinds of measurement situations. The first and the most important kind of situation is exemplified well by a generic SDT task. In this kind of task, a participant is required to respond to the stimuli presented on every trial and in random order by classifying each stimulus as belonging to one of two predefined classes. For example, it may be a perceptual task in which two different degraded stimuli are presented repeatedly and in random order, and the task is to decide which is which. This, in our view, is the simplest and at the same time the most powerful way of studying the process of ordinal response generation because the latent samples are intervened on, and repeated measurements make it possible to fit models much more realistic than would be possible if there was only one observation per participant and stimulus. That is but one reason that we believe it is not only justified but also quite useful to consider the remaining three kinds of situations as related to the first.

Another kind of situation is performing on an ability test composed of multiple items. This has more in common with the former kind of situation than may initially appear. To see why, compare the process of performing on an ability test comprising a fixed sequence of items to the process of performing on an SDT task that requires that words or non-words are classified as such. The methods are different in important ways but they are also similar in important ways, and the two kinds of response processes have some important things in common.

An ability test composed of dichotomous items is a special case of what in SDT is known as the single-interval task: a stimulus (here, a statement) is presented on every trial (here, once), and the task is to classify the stimulus as belonging to one of two predefined categories (here, as correct or incorrect). An ability test composed of two-alternative multiple-choice items is a series of pairs of alternatives such that each pair is composed of one correct and one incorrect alternative; in SDT, this kind of task is known as the two-alternative forced-choice task: two 
stimuli are presented on every trial, each belongs to a different class, and the task is to decide which one belongs to some reference class, e.g., which is bright (here, which one is correct).

In IRT, an ability test composed of dichotomous items could be represented by the Rasch model; in SDT, a randomized binary classification task could be represented by the Equal Variance (EV) Normal SDT model. Except for the presence of item parameters, about which we will have more to say later, there is little difference between the two models: The probability of the correct response according to the Normal Ogive Rasch model is given by $\Phi^{-1}\left(\beta_{n}-\delta_{i}\right)$, where $\Phi$ is the standard normal CDF, $\beta_{n}$ is the ability of person $n$, and $\delta_{i}$ is the difficulty of item $i$. The probability of the correct response of an unbiased individual according to the EV Normal SDT model that allows for the stimuli to differ in difficulty can be expressed as $\Phi^{-1}\left(\left(d_{n}^{\prime}-\delta_{n i}\right) / 2\right)$ where $d_{n}^{\prime}$ is the sensitivity of person $n$, and $\delta_{n i}$ is the difficulty of stimulus $i$ experienced by person $n$.

As noted by van der Maas et al. (2011), a self-report response, or, more specifically, its expected value, unless it is related to performance on some task, does not require any specific level of the attribute which it is supposed to be informative about. In other words, because a self-report response can always be systematically faked, by itself, this kind of response does not prove anything in the sense in which systematic above-chance performance on a task may prove something.

If certain conditions are met, both an SDT task and an ability test can prove something substantial. To see why exactly, first note that for certain types of tasks, not every expected pattern of response can co-occur with every level of the latent attribute. In a typical SDT task, for instance, above-chance performance cannot be systematically faked without cheating because, thanks to the random assignment of the stimulus class, performance can be systematically above chance only if the responses are influenced by the stimulus class. More generally, systematic abovechance performance on a randomized task cannot be faked, because the observed performance on such a task is an estimate of an identifiable causal effect that captures a part of what it means to be able to perform on that task.

Systematically faking good performance on a well-designed ability test with multiple-choice or dichotomous items is also unlikely exactly because of what this kind of test has in common with an SDT task. To minimize the probability of guessing, the items that comprise an ability test can be selected in a way that approximates the process of choosing the logical value of the alternatives independently from the order in which they appear, from what they are about, or from how they are phrased. Even when the order of items is fixed, if the correctness of the alternatives is hard to guess, the resulting ability test becomes a practical approximation of a randomized classification task. This similarity is of course also present when there are more than two classes of stimuli.

The intended purpose of an ability test with dichotomous items may be to measure an ability defined in one way or another, e.g., as knowledge of some subject matter or as intelligence. However, what this kind of test measures more directly is the ability to discriminate between two particular classes of items. From the perspective of a formal SDT model, correct and incorrect items in such a test are just two classes of stimuli, and nothing in an SDT model dictates that classification of stimuli as either correct or incorrect is excluded. From the perspective of a formal IRT model, correct and incorrect items are also just two classes of items, since all that is necessary and sufficient to interpret the response as either correct or not is 
the match or lack thereof between the response and the item. The interpretation of the items as either correct or incorrect is needed only when the ability to correctly discriminate between the two classes, which is what this kind of test measures more directly, is interpreted in terms of some underlying ability, which is what the test is supposed to measure.

Therefore, the main differences between some well-designed ability tests and some SDT tasks are that in an ability test, randomization is usually impractical even though it is desired, and, just like in a recognition test, because of memory, it almost never makes sense to collect more than one response to each item from the same individual. It follows that such an ability test is a special case of an SDT task, but the design is inherently weaker.

Finally, note that strictly speaking, the fundamental limitation of self-report has nothing to do with the lack of a relation between the observed response and performance on some task. Every self-report test is a task because the measured individual is instructed to respond to the items in a specific way. It is just that this task is not controlled by the person who administers the test, and so in general, it can be hoped, but it cannot be proved that the test instructions are followed to a sufficient degree.

One can say that some well-designed ability tests are like SDT tasks but without repeated measurement and strict randomization, and self-report tests of state are like SDT tasks but there is no randomization, and so the target of measurement is not an identifiable causal effect. Self-report tests of state (e.g., current mood) can be thought of as an intermediate case between an SDT task and a self-report test of a trait. The crucial features of the two kinds of self-report tests are that repeated measurements often make perfect sense and experimental manipulations that could potentially alter the target of measurement are often feasible in the case of tests that aim to measure a state-like variable, but not in the case of typical tests that aim to measure a trait-like variable.

Given the deep similarities between the four kinds of situations, we propose to view SDT models and some IRT models as either special cases or as generalizations of our model. The essential difference between IRT models and SDT models considered as statistical models is that there are item parameters in IRT models. Since the statistical part of our model was invented to get rid of item parameters whenever possible, we have to explain why we believe they should be introduced with caution.

In the following section, we argue that, even though the notion of item parameter and the notion of measurement invariance have a common origin, which is the recognition that the result of measurement and the target of measurement are not the same thing, the presence of item parameters makes it impossible to fully account for measurement bias. In particular, we argue that the presence of item parameters makes it impossible to account for one of the two universal sources of bias in the methods of measurement which can be naturally approximated by models that assume that a latent target of measurement manifests itself through a process that has a systematic part and a stochastic part, and that to account for this source of bias the role of item parameters needs to be reconsidered. We propose a causal definition of measurement invariance and we show that the commonly accepted statistical definition is flawed. Equipped with the new definition, in the remaining two sections of the paper, we try to show, by making use of all 
the essential features of the model that we propose, how this definition can guide the researcher in the process of establishing the validity of substantive conclusions.

On item parameters and measurement invariance

Here, we consider only unidimensional IRT models, but all that we say applies to models with more than one latent dimension as well as to Confirmatory Factor Analytic (CFA) models, as long as they are interpreted in terms of a psychological data generating process.

In a unidimensional IRT model, each individual is assumed to have a single unique latent value - a scalar attribute - which is constant across items. The relationship between the latent variable $L$ and the response variables $\boldsymbol{R}$ is typically characterized by two additional statistical assumptions: one is the monotonicity assumption; the other is conditional independence of $\boldsymbol{R}$ given $L$. The natural causal interpretation of such a model is that $L$ is the common cause of the response variables (Borsboom, Mellenbergh, \& Van Heerden, 2003). The relation between the latent variable and the response to a test item is described by the Item Response Function (IRF), which is a discrete parametric distribution of the response given the latent variable.

Certain parameters in IRT models, including the thresholds in the members of the cumulative family, may be item-specific, they may be specific to a group of people, but they are not person-specific, hence the name "item parameter". A reader who is new to IRT can see that this is the case by looking for IRT models with item parameters indexed by persons; to our knowledge, there are no such models in Van der Linden (2018), even though many different hierarchical IRT models, including hierarchical models with random effects in item parameters, are covered in this excellent and comprehensive book. There are also no such models in De Boeck (2004), even though this book, which we also think is excellent, is devoted to the so-called explanatory IRT models, characterized by the addition of predictors of item or person parameters.

There is a mundane, technical reason why item parameters sometimes have to be introduced: IRT models are typically used in situations when repeated measurement makes little sense. If there is only one observation per person and item, it is impossible, without very strong additional assumptions, to infer anything at all about the individual differences in the way of responding to individual items. This, however, is not the reason usually put forward by the proponents of IRT. For instance, as Van der Linden (2018) states in the preface to the seminal Handbook of Item Response Theory (emphasis is ours):

The major breakthrough it [IRT] presents is the solution to one of the fundamental flaws inherent in classical test theory - its systematic confounding of what we measure with the test items used to measure it. [...] IRT solves the problem by recognizing each response as the outcome of a distinct probability experiment that has to be modelled with separate parameters for the item and test taker effects. (p. xvii)

What is here proclaimed as solved is the conceptual problem associated with Classical Test Theory (CTT, see Borsboom, 2005, for a lucid explanation): In CTT, the true score is defined as the expected value of the observed score. If the true 
score thus defined is interpreted as what the test is supposed to measure, then the target of measurement becomes something that is defined, statistically, in terms of the method. According to this definition, for example, the level of intelligence of a particular person is the true mean of the intelligence test score of that person. This is known to lead to some serious issues (Klein \& Cleary, 1967; Lord \& Novick, 1968; Lumsden, 1976).

However, from the mere recognition that the method of measurement and the target of measurement is not the same thing, it does not yet follow that measurement models must have item parameters. It also does not follow that an introduction of a statistical notion, which the notion of item parameter is, solves anything other than a statistical problem.

Once the difference between the target of measurement and the method of measurement is recognized, we are immediately faced with the possibility that the results of measurement may be systematically misleading, i.e., with the possible lack of measurement invariance. In IRT, a lack of measurement invariance is called item bias. According to the commonly accepted definition (Angoff, 1993), which is also statistical, an item is biased with respect to some variable, $V$, that represents group membership, when the distributions of response, $R$, to the item, conditional on the value of the target of measurement, $T$, differ between values of $V$, i.e., when $p(R \mid T) \neq p(R \mid T, V)$. When that is the case, the differences in performance on the test can be attributed, at least in part, to differences that may have nothing to do with the target of measurement.

Realizing that a method of measurement may be biased is critically important in research because it implies that what is being actually measured may not be the target, or at least not only the target, and so the observed statistical relations between the measurements and anything else may be due to some unforeseen or unintended causes. Measurement bias may also be important in an applied setting, where it can lead to, e.g., unfair treatment of some group.

The literature on item bias is already so vast that there is an excellent book on the subject. As Millsap (2012) notes in the preface to this book:

One reason for my fascination with the [item bias] detection problem is that it remains only partially solved. Fundamentally, while we have detection methods that work under limited circumstances, we have no completely general method for detecting bias in psychological measures that works across all of the many measurement conditions encountered in psychology. [...] A further problem is that it is usually hard to verify some of the assumptions that are needed in practice for many of the detection methods. (p. ix)

Note that even though the commonly accepted definition of item bias is statistical, absent any causal assumptions about the possible sources of bias, such as that an item is phrased using words that may be understood differently by different groups of people, there is nothing that can guide the researcher in looking for evidence of a lack of measurement invariance. The necessary step then in addressing measurement invariance is recognizing that it is a causal problem. To his credit, Millsap explicitly acknowledges that the problem is causal, but so far, at least to our knowledge, only van Bork et al. (2020) have approached this issue from the causal-theoretic perspective. 
Based on the examples of measurement bias that can be found in the literature a causal definition of measurement invariance can be derived. Let $R$ be the result of measurement and let $T$ be the latent target of measurement. $R$ is biased as a measure of $T$ with respect to a manifest variable $V$, where $R \neq V$, if there exists an active (i.e., one without a collider, see, e.g., Pearl et al., 2016) causal path between $R$ and $V$ such that $T$ is not a node on this path and this path does not emanate from $R$. For brevity, we will call such paths "bias-inducing". When a biasinducing path exists between $R$ and $V, T$ does not block it (see the d-separation criterion Pearl et al., 2016), and so $R$ and $V$ may be dependent given $T$, just as the statistical definition requires. Note that this definition does not require that $R$ is valid in the sense proposed by Borsboom, Mellenbergh, and van Heerden (2004); in particular, it does not require that $T$ exists.

It is now easy to give examples of situations when the commonly accepted statistical definition fails. For instance, if $T \rightarrow R, R \rightarrow V$, and $R$ has no systematic causes other than $T$, then $R$ and $V$ may be dependent given $T$, even though it does not make sense to say that $R$ is biased in this case. The statistical definition fails because it does not say anything about the causal relations between $R$ and $V$. In particular, it does not say anything about what manifest variables (or criteria of group membership, as they are usually thought of) can or cannot be considered.

Another counterexample is when $R$ and $V$ both influence $T$. This can happen if feedback is provided after the response to every item on an ability test, thus facilitating learning $(R \rightarrow T)$, but some people $(V)$ pay less attention to feedback or they do not trust it as much as others $(V \rightarrow T)$. In that case, $R$ and $V$ may be dependent given $T$ because of collider bias, but this of course does not yet mean that $R$ is biased.

To see if the causal definition works it is worth considering some examples, some special cases, and some of its implications. One special case is when $R$ correlates with some manifest variable but it is not influenced by $T$, either because the method is poorly designed or because $T$ does not exist. It makes sense to say that $R$ is biased as a measure of $T$ in either case because $R$ measures something, and even when the target does not exist, it makes sense to say that such a measure is systematically misleading about the target. Another special case is when $R$ is completely random. Regardless of whether the target exists or not, such a measure is not biased with respect to anything, because even though it is misleading about the target, it is not systematically so.

To see if the causal definition captures the intended meaning consider how it matches an example given by Millsap (2012), which is a hypothetical faulty scale that gives consistently different results $(R)$ for objects that have the same weight $(T)$ but that differ in shape $(V)$ : In that case, $p(R \mid V, T=t) \neq p(R \mid T=t)$ for some $t$ because for some pair of objects of weight $t$ with shapes $v_{1}$ and $v_{2}$ we have that $p\left(R \mid V=v_{1}, T=t\right) \neq p\left(R \mid V=v_{2}, T=t\right)$. This has to be because of how the shape of an object affects the scale without affecting the weight of the object, i.e., because of $V \rightarrow R$, or because the readings on the scale and the shapes of objects have at least one common cause which is not $T$ and which does not influence $T$, i.e., because of $R \leftrightarrow V$.

An important consequence of this definition is that, unless $R$ responds immediately to changes in $V$, for a bias-inducing path between $R$ and $V$ to exist, there has to exist some third variable $N$ such that $N$ is a node on this path. This "nuisance" variable is part of a reason that $R$ is biased. For instance, a group of people could 
understand an item on a personality test differently than the rest of the population. This possibility could be naturally represented in the model as a difference in the sampled dimension. As implied by the causal definition of measurement bias, the common way of accounting for item bias is to add a latent nuisance variable to the model (Ackerman \& Evans, 1994; Camilli, 1992; Mellenbergh, 1989). Importantly, however, the differences in the underlying dimension are one universal source of measurement bias, but they are not the only such source.

Another intuitive consequence of the causal definition, but not of the statistical definition, is that, in general, the more indirect the method of measurement is, i.e., the further the target of measurement is from the response, the more opportunities there are for measurement bias to exist. That is because any node $N$ on the path from $T$ to $R$ may have some observable consequence $V$ such that $R$ and $V$ may be statistically dependent given $T$.

It also follows from the causal definition of measurement bias that most, if not all, methods of measurement of latent psychological variables are biased, probably in many different ways. The reason is that, unlike in the case of some methods of physical measurement, the error in measurement of a latent psychological variable cannot be thought of as random noise. For instance, being tired may influence how a person performs on an intelligence test, and tired people may move slower on average, and so intelligence tests may be, and to some extent probably are, biased against people who move slowly. That is but one reason that, in general, a correlation between reaction time and performance on an intelligence test cannot be interpreted solely in terms of the effects of intelligence. Yet another consequence of the causal definition is that, contrary to what some proponents of IRT claim, measurement invariance cannot be established by observing a good fit between any particular statistical model, including any IRT model, and the response data (see Asûn, Rdz-Navarro, \& Alvarado, 2017, for another reason that this cannot be the case).

The most commonly used item bias detection methods, i.e., Differential Item Functioning tests (Zumbo, 2007; Holland \& Wainer, 2012; Osterlind \& Everson, 2009), target statistically defined group-level effects. It may be that item bias is usually thought of in IRT as a group-level effect because item parameters are by definition group- or population-level properties of items, but the former does not follow from the latter. To see why simply note that neither the notion of measurement model nor the notion of item parameter plays any role in the statistical or the causal definition of measurement bias. Moreover, it is clear that if items can function differently for different groups, then they can function differently for different individuals or for the same individual in different situations. And yet, even though the possibility of individual item bias is by no means ignored - it underlies all methods for appropriateness measurement or for the assessment of person fit (Drasgow, Levine, \& Williams, 1985; Meijer \& Sijtsma, 2001) - this problem, as well as the methods designed to address it, seems to play a minor role in contemporary psychometrics. Because of that, what may be another universal source of measurement bias in psychology, which are inter- and intra-individual differences in the systematic part of the mapping between the latent dimension and the response, remains largely neglected.

Consider the quintessential item parameter, which is the difficulty of an ability test item. For example, in the case of a very difficult item, only 1 percent of the population may be thought to have an above-chance probability of providing the 
correct answer. The value of this parameter is by definition a property of some population or a group, not a property of an individual. Undoubtedly, estimates of item parameters say something important about items, and in applied settings, e.g., in prediction or in diagnosis, it may be very useful to know how difficult items are in that sense.

However, if item parameters represent statistical properties of groups of individuals, then they cannot account for inter- and intra-individual differences in the way of responding. If they cannot account for inter- and intra-individual differences in the way of responding, then they cannot be given a realist interpretation in terms of the properties of the psychological data generating process. The causal gap between the latent variable that is supposed to be measured by a test and the response to an item on that test cannot be filled by introducing a simple item response function: It is impossible for an item to have the property that the target latent psychological trait or state, such as intelligence or current mood, manifests itself stochastically through the process of responding to that item in the same way for every pair of individuals in some group, or for a particular individual in every situation.

Mixing causal and statistical notions requires great care; because of limitations of study design and current knowledge, many unrealistic simplifying assumptions have to be introduced to estimate approximations of target quantities, but issues of identifiability are not an excuse for working with flawed definitions of the variables of interest. Identifiability is something to worry about when a statistical model is to be fitted to data, not when the target quantities are defined. Otherwise, the target of measurement is once again defined in terms of the method, and the researcher is left with an often-impossible task of finding out what the results obtained by using an ad hoc method mean (see Borsboom, 2005, for a similar observation about how test construction and validation is usually approached).

It is the person, not the item, that does the categorisation leading to the response. Number of words is clearly a property of an item, as is the property of being expressed in the form of a statement or a question, but those are known constants; the difficulty of an item understood as something that should not be confused with the ability to guess the correct answer is a different kind of property. It cannot merely be a constant property of an item because it depends on an unobservable psychological process, and to our knowledge, no constant properties of such processes have so far been discovered. From that point of view, the presence of item parameters in a measurement model is an extreme simplification.

When the true item effects are defined in terms of qualitative causal relations, there is no reason to simplify anything. If we accept the simple fact that, e.g., item difficulty or probability of guessing can differ between as well as within individuals, then the true item effect becomes the mean of the distribution of situationand-person-specific item effects. As we illustrate later, in a nonlinear model, an item effect thus defined cannot be captured well by an item parameter regardless of sample size. It follows that estimates of item parameters are either poor approximations of average item effects or they are estimates of purely statistical properties.

In the remaining part, we show what can be achieved when an SDT model, interpreted as a causal measurement model, does not have item parameters, what problems may arise when it does, and how such problems can be addressed. 


\section{A causal reintroduction to SDT models}

According to the common interpretation of an SDT model, when the stimulus is presented, an unspecified decision process generates an internal evidence sample $s$, which is usually assumed to be normally distributed with variance fixed at unity to make the model identifiable. The individual is assumed to classify the stimulus as belonging to the second stimulus class (e.g., "signal" instead of "noise", or "new" instead of "old", or "word" instead of "non-word") if and only if $s>\tau$, where $\tau$ is the main decision threshold, also known as the decision criterion.

In addition to the binary classification response, the participant may also provide a rating, such as a confidence rating. In such cases, it can be assumed that the ratings are just like binary classification responses, but they correspond to different internal decision thresholds. For instance, responding "I am almost certain that it was a word" may be assumed to be just like responding "it was a word", except that the internal evidence sample must have a high enough value to warrant near-certainty. The two kinds of response can then be thought of as one kind of response: According to the model, the rating $k$ is provided if and only if $\tau_{k-1}<s \leq \tau_{k}$, where $\tau$ is the vector of $K+1$ ordered thresholds such that $\tau_{0}=-\infty$ and $\tau_{K}=\infty$, meaning that only $K-1$ thresholds are free to vary. As the reader may have already noticed, this is just a cumulative model of the kind presented in Fig. 2, but with a normally distributed latent state variable.

So far, we have introduced one statistical assumption, i.e., that $s$ has a normal distribution with unit variance, but even this assumption can be, and often is, causally motivated, e.g., by stating that the decision process may integrate information and accumulate noise from many different sources, and so it follows from the central limit theorem that a normal distribution is a natural first approximation (see, e.g., Wickens, 2002).

What is perhaps easier to miss is that we have also either introduced a strong causal assumption about the way the motor response is generated or we have accepted a rather special definition of sensitivity: In an SDT model, the observed response is a deterministic function of $s$ and $\tau$, meaning that once the values of both parameters are fixed, the decision is completely determined, and the motor response is identified with the decision. Clearly, participants cannot execute every motor response without error, and so this property of an SDT model may sometimes be problematic.

Most importantly, in this kind of model, it is assumed that the mean of the latent evidence distribution, but not the thresholds, can be influenced by the stimulus class. When this happens, the evidence samples are higher or lower on average, depending on what kind of stimulus is presented. This causal effect makes discrimination possible. An example of this kind of model is shown in Fig. 3 below. 

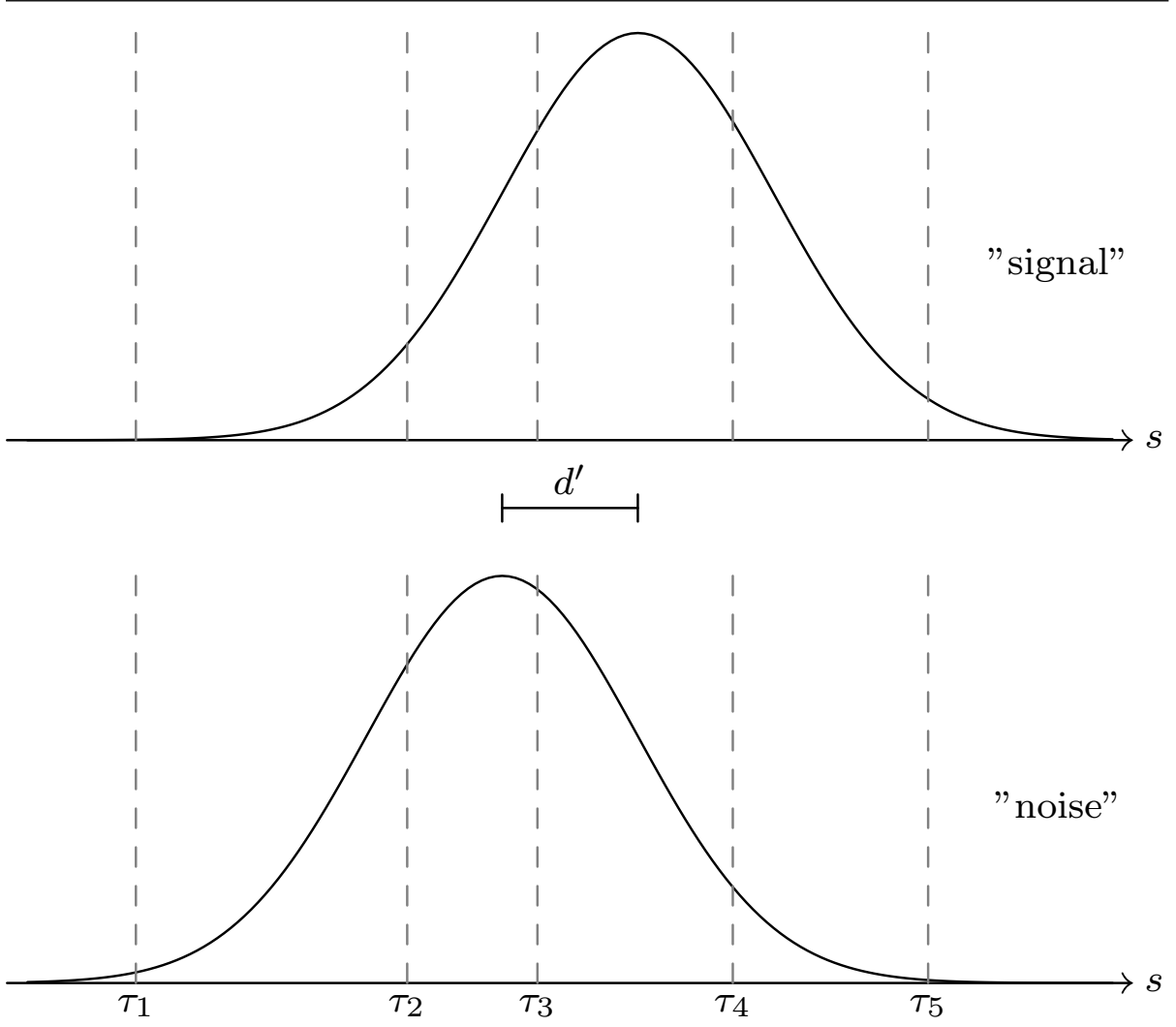

Fig. 3: The Equal Variance Normal Signal Detection Theory model with multiple thresholds. The upper (lower) density curve is the distribution of the internal evidence samples when the stimulus is of class "signal" ("noise"). Sensitivity $\left(d^{\prime}\right)$ is defined as the distance between the means of the two distributions.

The assumption that the stimulus class does not influence the thresholds is necessary but not sufficient if we want to interpret the estimates of threshold parameters as estimates of actual decision thresholds. If evidence was found that the value of a threshold parameter is influenced by the stimulus class, it could mean (but it does not have to, as we later explain) that this parameter captures at least some sensitivity, which it is not supposed to.

The causal assumptions of the model in its most basic form can then be formally represented by a simple causal graph: $S \rightarrow R \leftarrow \tau$, where $S$ is the stimulus class. Since in a typical SDT study, the stimulus class is chosen at random, it is impossible for anything to influence the stimulus class. That is why the arrows pointing to $S$, but not to $R$ or $\tau$, can always be safely omitted. However, in a sense, it is the arrows pointing to $R$ or $\tau$ that are more important because they may be a part of a bias-inducing path.

The assumptions represented by this causal graph alone have a special status. On the one hand, when supplemented with statistical assumptions, they become a formal part of a testable causal model. On the other hand, they can be inter- 
preted as a simultaneous weak definition of the terms "sensitivity" and "decision criterion" or "decision threshold". That is because, regardless of the quantitative details of the decision process, a person cannot be said to be sensitive to the stimulus class if the stimulus class does not somehow influence that person, and for something to play the role of the decision criterion that something must influence the response but it cannot itself be influenced by the stimulus class ${ }^{2}$.

The two formal definitions are too weak if certain stronger interpretations of the term "sensitivity" are desired. For example, a person may trade accuracy for speed or may not pay as much attention to the task as would be otherwise possible, in which case the true causal effect of $S$ on $R$ will not represent the maximum attainable sensitivity. When such strategic or motivational factors are treated as confounds or as sources of measurement bias, it is because something resembling maximum attainable sensitivity is the target of inquiry.

Moreover, sensitivity defined as the total causal effect of $S$ on $R$ may have nothing to do with the ability to discriminate between the stimulus classes. For instance, according to the UV SDT model, the two internal evidence distributions are both normal, but they may differ in variance. When they differ in variance but not in mean (i.e., $\left.d^{\prime}=0\right) S$ does influence $R$, because in that case $p(R \mid S=1) \neq$ $p(R \mid S=2)$, but this kind of causal effect does not make discrimination possible.

Another, already mentioned, problem with the definition of sensitivity as the causal effect of $S$ on $R$ is that this effect depends not only on the ability to mentally discriminate between the stimulus classes but also on the ability to correctly express the decision by e.g., pressing the response key that corresponds to the mentally chosen stimulus class. Making the definition narrower by assuming that sensitivity is not the total causal effect, but just the effect in means, does not solve the problem of effects at the motor stage. If mental discrimination is the target of inquiry, effects at the motor stage may confound the results.

Finally, there is nothing in the definition of sensitivity as an arrow from $S$ to $R$ or as $d^{\prime}$ that captures the purposeful nature of classification. As a result, this definition is not psychological; it only captures a part of the causal part of the psychological meaning of the term. To see why, note that this definition applies just as well to an arbitrary mindless device, such as a thermostat, that differently and in a fixed way responds to two different kinds of input. The same can be said about the decision threshold(s) - at the causal diagram level, those are arbitrary properties of the distribution of an arbitrary outcome that do not depend on the type of input. The purposeful aspect could perhaps be captured by making use of a rational theory (Anderson, 2013; Chater \& Oaksford, 1999; Oaksford \& Chater, 1998), but this is not something we can properly address in this paper.

Despite the conceptual limitations stemming from its simplicity, there is good reason to believe that an SDT model may at least help separate the effects in sensitivity from the effects in the decision threshold(s), which is something that cannot be said about the percent-correct score. For example, if an experimental manipulation in a study on recognition affects the tendency to classify the stimuli as old, which means that it affects the main decision threshold, then it may also affect the percent-correct score. To see why imagine that presenting an old (new) stimulus shifts the latent mean to the left (right) in both conditions - this is the sensitivity effect. Imagine also that there is just one threshold, on average it

2 That is not to say that the thresholds cannot be influenced by the previously classified stimuli 
is in the neutral position in the control condition, and that in the experimental condition, a change of stimulus moves the latent mean further from the midpoint between the two latent distributions (higher sensitivity), but the threshold is also on average moved far to the left from the midpoint. Because of the threshold effect, the great majority of stimuli are classified as old and, if there is an equal number of old and new stimuli, the expected percent-correct score may be lower in the experimental condition even though sensitivity is higher, leading to conclusions that are the exact opposite of the truth (see Liddell \& Kruschke, 2018, for another example of this kind of effect, but based on a model with item parameters).

If the intent of the researcher is to estimate the causal effect of the experimental manipulation on the ability to recognize the previously presented words, then an SDT model is a measurement model, albeit of the kind that is fitted to the data whenever it is used. Since all randomly assigned variables are manifest variables, this is an instance of measurement bias: The conditional distributions $p\left(R \mid d^{\prime}\right)$, where $R$ is the percent-correct score, differ between study conditions, which means that as a measure of the ability to recognize the words the percent-correct score is biased in a way that is problematic in this case. If it is surprising that the notion of measurement bias is so closely related to the issue of the validity of a substantive interpretation of the results of an experiment, note that a measurement obtained by administering a test is a response, i.e., it is a sample from the interventional distribution $p(R \mid d o($ test $))$.

Note also that only the causal definition of measurement bias can be used to formally express the source of the problem: There exists a nuisance variable $\tau$ (the decision threshold) and a manifest variable $V$ (the experimental manipulation), such that $R \leftarrow \tau \leftarrow V$. Incidentally, this is also a realistic, even if somewhat extreme, example of a commonly occurring violation of the monotonicity assumption, but not of the local monotonicity assumption. Because sensitivity is now by definition a causal effect and by virtue of it being causal it captures at least part of the intended psychological meaning, there is also another, formally justified and more direct way of describing this situation: there is a systematic threshold effect that changes the psychological meaning of the statistical percent-correct score effect.

\section{Statistical and causal testing of a false SDT model}

All the analyses described in this paper were performed using the package for $\mathrm{R}$ (R Core Team, 2017), called bhsdtr2, that uses the state-of-the-art Stan sampler (Carpenter et al., 2016) to fit the models. The $\mathrm{R}$ script that was used to perform all the analyses and produce all the plots is available at the bhsdtr2 GitHub repository https://github.com/boryspaulewicz/bhsdtr2. In all the analyses, we used a subset of the dataset, called gabor, that comes with the bhsdtr2 package.

The participants in this unpublished study had to classify a briefly presented Gabor patch as tilted left or right using the arrow keys. They were also asked to rate the stimuli on the Perceptual Awareness Scale (Ramsøy \& Overgaard, 2004) with four possible ratings that ranged from "no experience" to "absolutely clear image". Since in this case there were 4 ratings, the compound response had 8 possible values, ranging from $1=$ tilted left + absolutely clear image, through 4 $=$ tilted left + no experience, to $8=$ tilted right + absolutely clear image. The 
Gabor patch was immediately followed by a mask. To simplify the presentation, we only used the data from the $32 \mathrm{~ms}$ presentation time condition in which 29 participants provided the ratings after the binary classification decision.

In order to interpret the estimates of the model parameters in terms of meaningful properties of the data generating process, we have to introduce additional causal assumptions that are specific to the design of this study. Those are worth listing to further emphasize just how approximate the model is. Since there are multiple measurements, we have to acknowledge the fact that by fitting a model with one sensitivity, one set of thresholds, and no other dependency structure, we are assuming that the process is essentially stationary, i.e., that it is so stable that we can ignore all other kinds of inter-trial variability or dependence, such as would result from learning, fatigue, other changes in the way the task is approached, or from the operation of external factors. Finally, not only are there multiple measurements per participant but there are also multiple participants. Since it is a causal model of the response process, we naturally assume that each participant has a unique sensitivity and a unique set of thresholds.

Initially, we fitted an SDT model that accounted for participant-specific sensitivity and thresholds. It was the same model as the one described in Paulewicz and Blaut (2020), except that it used a different, more convenient link function, which is called "log_distance" in bhsdtr2. This and other similar link functions are described in the Appendix - all that matters for our immediate purposes is that an order-preserving link function makes it possible to account for arbitrary effects in the pattern of the thresholds without violating the assumption that the thresholds are ordered. Individual levels of sensitivity were represented as random effects (in the $\log \left(d^{\prime}\right)$ space, see Paulewicz \& Blaut, 2020, for why this is important) and individual thresholds were represented as correlated random effects (in the space implied by the link function).

Seven chains of 5000 iterations each were run simultaneously; first 2000 posterior samples were discarded. The chains exhibited good mixing and seemed to have converged; there were more than 5000 effective posterior samples of each fixed effect, more than 9000 posterior samples of each random effect, and none of the Gelman-Rubin statistics crossed the 1.01 threshold.

The fit of an SDT model is usually assessed by means of the Receiver Operating Characteristic (ROC) plot (Alsop, 1998). An ROC plot shows how the probability of "hit" $(p(H))$, which is the probability of responding "2" to the stimulus of class "2" (often called "signal"), depends on the probability of "false alarm" $(p(F))$, which is the probability of responding "2" to the stimulus of class "1" (or "noise"). Given the model, the two quantities are two independent sources of information that together correspond to unique values of $d^{\prime}$ and $\tau$. The first (second) probability is equal to the area under the density curve in the lower (upper) panel of Fig. 3 to the right of the main decision threshold, i.e., $p(H)=1-\Phi\left(\tau-d^{\prime} / 2\right)=\Phi\left(d^{\prime} / 2-\tau\right)$ and $p(F)=1-\Phi\left(\tau+d^{\prime} / 2\right)=\Phi\left(-d^{\prime} / 2-\tau\right)$, if the middle point between the latent distributions is the origin of the scale.

When $d^{\prime}$ is fixed, shifting the main decision threshold to the right results in a lower probability of false alarm at the cost of a lower probability of hit. By tracing the possible positions of the main decision threshold from minus to plus infinity, a continuous ROC curve that corresponds to the fixed $d^{\prime}$ value is obtained. A ROC plot can be used to assess model fit by comparing the ROC curves implied by the fitted model to the observed pairs of proportions of hits and false alarms 
obtained by treating all categories of response lower than $l$ as response "1", and all categories of response greater or equal than $l$ as response " 2 " for $l=2, \ldots, K$.

When the relationship between $p(H)$ and $p(F)$ is plotted in the $z$ space, i.e., as $\Phi^{-1}(p(H))$ vs. $\Phi^{-1}(p(F))$, the ROC curve becomes a straight line. This line has a unit slope and an intercept that is equal to $d^{\prime}$ because $\Phi^{-1}(p(H))=d^{\prime} / 2-\tau=$ $-d^{\prime} 2 / 2-\tau+d^{\prime}=\Phi^{-1}(p(F))+d^{\prime}$. It is just as easy to show that when the two latent distributions have different variances, which they are allowed to in the UV SDT model, the slope of the lines in the zROC plot is different from 1. Individual ROC plots that show how the model fitted to the gabor dataset are presented in Fig. 4 below.

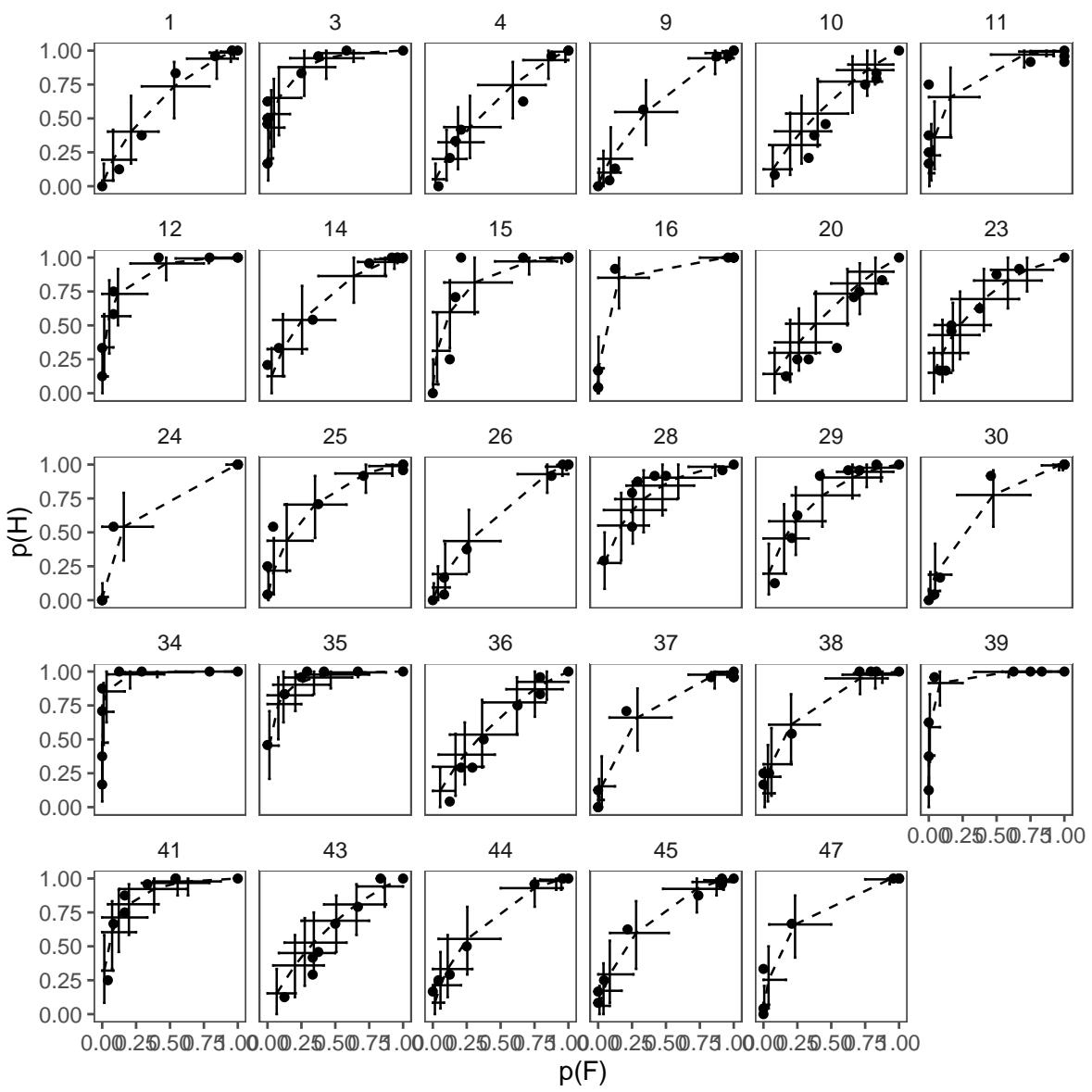

Fig. 4: Individual ROC plots with $95 \%$ predictive intervals: every panel shows the implied ROC curve; the points represent the observed proportions of hits and false alarms. See the description in the text for more details. 
Another way of assessing the fit of the statistical part of this model is by looking at what we call Response Distribution (RD) plots. Those are plots comparing the implied and the observed frequencies of ratings. RD plots have some advantages over ROC plots. In contrast to ROC plots, they do not require two-dimensional regions to represent the uncertainty, and since they do not represent cumulative probabilities, they are not as smoothed as the ROC curves, which may sometimes make a lack of fit more apparent on an RD plot. Finally, such plots are applicable in situations when ROC plots are not, for instance, in observational studies, because they do not require that there are two classes of stimuli and two classes of matching responses.

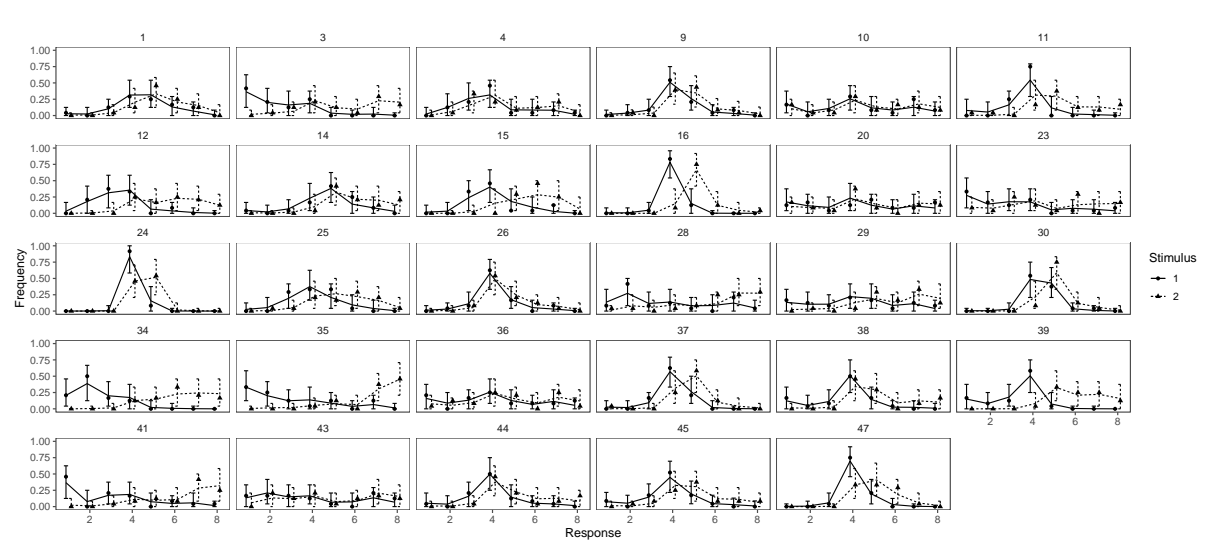

Fig. 5: Individual Conditional Response Distribution plots with $95 \%$ posterior predictive intervals: every panel shows, as lines, two implied distributions of responses of one participant, one for the first, and one for the second stimulus class; the points represent the observed proportions.

As can be seen in Figs. 4 and 5, the fit of the model was good even at the individual level. This indicates that there seems to be no reason to strongly reject the statistical assumptions of the model. Note that the reason that this SDT model could be statistically tested is that the data come from a rating experiment: If the response was binary there would be only one decision threshold per participant, and the model would fit perfectly because it would be saturated (the number of free parameters would be equal to the number of degrees of freedom in the data). Note also that a good fit was to be expected, since there were only 6 out of 14 degrees of freedom per participant left in the data ${ }^{3}$, and a great number of studies have shown that SDT models often fit this kind of data well (see, e.g., Wickens, 2002; Macmillan \& Creelman, 2004).

However, unless a statistical test is specifically designed to answer a causal query, the fact that a model, as a statistical model, describes the data well, or that its predictive performance is better than that of some other model, is not

3 Once the proportions of $K-1$ rating categories for each stimulus class are known, the proportion of the remaining category is also known, and the participant-specific sub-model has $K$ free parameters: 1 sensitivity and $K-1$ thresholds. 
in itself evidence that the assumed interpretation of the parameters in terms of meaningful properties of the data generating process is valid.

Many important causal assumptions cannot be tested in this case. For instance, because there are only 48 trials per participant, without strong additional assumptions, it is impossible to test if the decision thresholds, the latent mean, or the sensitivity did not vary between trials, although it is obvious that all those quantities cannot possibly remain exactly the same during the experiment. There does not seem to be enough trials to directly test, by fitting, e.g., the diffusion model, if the estimates are not contaminated by time-accuracy trade-off effects, but it is safe to assume that, to an unknown degree, they are.

However, given that there are multiple measurements and the stimulus class is randomized, we can test one causal assumption: that the stimulus class does not influence the thresholds. This is where an SDT model, when interpreted causally, implies no statistical effect. That this particular prediction follows from the central causal assumption of the model makes the test particularly relevant to partially establishing the psychological meaning of the estimates.

To test for the lack of selectivity of influence of the stimulus class, instead of assuming that the threshold parameters represent the actual decision thresholds, we have to temporarily allow for the possibility that they do not. This requires a more general model, one in which the threshold parameters can be influenced by the stimulus class. The resulting model may seem strange to those familiar with Signal Detection Theory, but the influence of the stimulus class on the threshold parameters is not made impossible by the study design.

In this way, we exploit an important property of all cumulative models, i.e., the relation between the thresholds and the latent mean. Once the type of the latent distribution is fixed (e.g., normal with unit variance), a change in the latent mean is indistinguishable from a uniform shift in the thresholds in the opposite direction. On the one hand, this means that when arbitrary effects in the thresholds are allowed, the latent mean parameter is descriptively redundant: Unless there is some additional source of information, there is no way of knowing if the effect is due to the change of mean or due to the change of thresholds. On the other hand, however, this means that a uniform shift of all the thresholds in the same direction can be interpreted as a change of the latent mean in the opposite direction. If this is an effect of a randomized stimulus class, there is nothing arbitrary about this interpretation.

To test if the shift was uniform, we fitted a hierarchical cumulative model that assumed that each participant has two sets of $K-1$ thresholds, one for each stimulus class, and that the latent samples have a normal distribution with mean fixed at 0 and variance fixed at 1 . The participant-specific thresholds were modelled as correlated random effects in the space implied by the link function. This model is saturated with respect to the aggregated data, and so it has to fit perfectly, but there is still uncertainty associated with the estimates of its parameters.

Seven chains of 5000 iterations each were run simultaneously, and the first 2000 posterior samples were discarded. The chains exhibited good mixing and seemed to have converged; there were more than 10000 effective posterior samples of each fixed effect, more than 10000 posterior samples of each random effect, and none of the Gelman-Rubin statistics crossed the 1.01 threshold.

The point estimates of the differences between the middle thresholds correlated strongly $(r=.94)$ with the point estimates of sensitivity from the SDT model fitted 
earlier. This shows that as long as the increase in model complexity is acceptable, the latent mean parameter can sometimes be omitted.

To assess apparent non-selectivity, we calculated the following contrasts: $\boldsymbol{x}_{i, k, s}=$ $\boldsymbol{\tau}_{i, k, 1, s}^{*}-\boldsymbol{\tau}_{i, k, 2, s}^{*}$ where $\boldsymbol{\tau}_{i, k, j, s}^{*}=\boldsymbol{\tau}_{i, k, j, s}-1 / k \sum_{k}\left(\boldsymbol{\tau}_{i, k, j, s}\right), i$ is the participant index, $k$ is the threshold index, $j=1,2$ is the stimulus class, and $s$ is the posterior sample number. In words, we removed any uniform effects by centring the posterior threshold samples on the average threshold position. Then, we subtracted the centred posterior samples (the $\tau^{*}$-s) of thresholds for the "left" stimulus from the cantered posterior samples of thresholds for the "right" stimulus. The resulting contrasts allowed us to test if the distances between the corresponding thresholds for the two stimulus classes match. This way, we tested for any changes in the pattern of thresholds' placement due to change of the stimulus class, not for changes in their average position which correspond to sensitivity effects.

The 90\% Highest Posterior Density (HPD) intervals for the differences in the thresholds' patterns are shown in Fig. 6 below. We used slightly more conservative $90 \%$ intervals instead of the more common $95 \%$ intervals because those are estimates of interactive random effects in a relatively complicated model, and there are only 48 trials per participant per condition. The narrower intervals are more conservative in this case because the model implies no effect in the estimated contrasts.

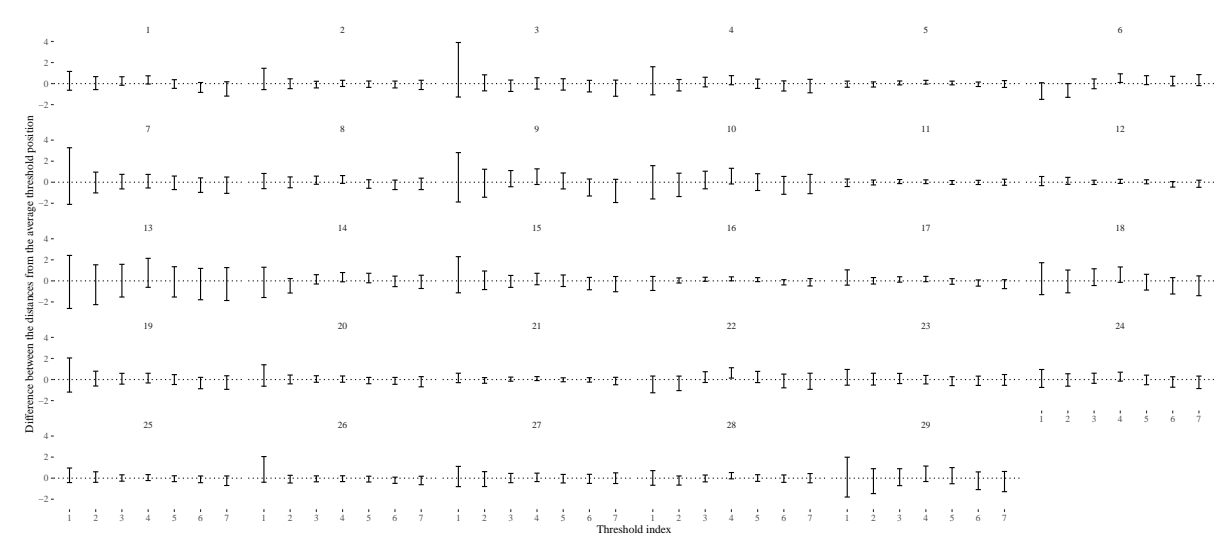

Fig. 6: Estimates of the differences in the pattern of the thresholds for the first and the second stimulus class. The vertical bars are $90 \%$ HPD intervals. Every panel shows the interval estimates of contrasts for one participant. See the text for more details.

Only 2 percent of the $90 \%$ HPD intervals did not contain zero, which can be interpreted as a lack of evidence that the patterns of thresholds' placement were different. Interestingly, even though for some of the participants, the stimulusspecific patterns seem very similar, for some, the differences appear to assume an inverted U shape. This kind of shape is possible if the UV SDT model is true. Hence, like a zROC plot, this kind of test can be used to look for evidence that the distributional assumptions may not be appropriate. 
It seems worth listing the statistical features of our model that, together with the fact that it was defined as a causal model, made it possible to test this one causal assumption. Firstly, even if the effect of the stimulus class was far from selective at the individual level, the group level pattern of thresholds could correspond to an approximately constant shift. Because the tested causal assumption concerns the way the data generating process works in every individual and in every context, it is important that this assumption is tested at the participant $\times$ condition level. Secondly, the fact that the thresholds were flexible but ordered (theoretically justified constraints) and modelled as correlated random effects (pooling strength) may have improved their estimates. Finally, Bayesian inference allowed us to calculate interval estimates of arbitrary contrasts, which made it possible to directly assess the conditional dependencies ruled out by the causal assumption of selective influence.

\section{The consequences of ignoring individual differences in the thresholds}

To our knowledge, the model proposed in Paulewicz and Blaut (2020) was the first cumulative model that allowed for arbitrary effects in individual thresholds while preserving their order. To illustrate what happens when such effects are ignored, i.e., when the thresholds are item parameters, we have again fitted two models, but this time to the simulated data.

We have simulated 100 responses per stimulus class per participant from the SDT model fitted earlier using posterior means as known true values. The number of trials per participant was increased from 48 to 100 because this way, the influence of the default priors, which were already only weakly informative, was made weaker still, but the sample size was not unrealistically big. The two fitted models were the true model from which the data were simulated, and the model that had item parameters in the sense that it assumed that all participants have the same pattern of thresholds' placement.

For both models, seven chains of 5000 iterations each were run simultaneously, and the first 2000 posterior samples were discarded. The chains exhibited similar mixing performance as in the first analysis.

The estimates of sensitivity and thresholds obtained in the first analysis were within the range of values typically observed in similar studies. Thus, the fitted model from which the data were simulated can be thought of as a simplified but somewhat realistic representation of individual differences in sensitivity and thresholds.

Because we know where the data came from, we also know that the simplified model cannot fit the data well at the individual level, and so even if the fit was good at the aggregate level, it would only be good by accident. It is pointless to try to find evidence of the existence of bias in point and interval estimates of individual thresholds in the simplified model: by design, those parameters do not correspond to any meaningful property of the data generating process; because of non-linearity, the relationship between the estimates of the overly-constrained threshold parameters and the (known in this case) properties of the data generating process is anything but simple. The sensitivity parameters in the simplified model were not overly constrained, but since both kinds of estimates are interdependent, they cannot possibly be unbiased either. However, it is instructive to see just how 
bad they are and, more importantly, what can or cannot be done about this kind of problem in a real setting, i.e., when the true values of the target causal quantities are not known.

Assessing the magnitude of causal estimate bias

Firstly, note that from a Bayesian perspective, because of the influence of priors, unbiasedness as such is often not important. Here, we are interested in unbiasedness in the limit of large samples, i.e., in asymptotic unbiasedness, which, unless the bias is negligibly small, is important. Secondly, we are not interested in estimate bias from a purely statistical perspective but from a causal perspective. We want to know if bias is such that it leads to invalid conclusions about meaningful properties of the data generating process, i.e., about the source of the data, not about the distribution of the data.

We propose to approach this problem in a way similar to how we assessed estimate bias in Paulewicz and Blaut (2020), which was simply to roughly assess the coverage of the credible intervals obtained by fitting a model that was known to be false in a specific way. This time, however, we can provide a causal interpretation of this procedure.

If a model does not account for a source of variance that is present in the data, such as an effect of participants in a repeated measures design, then the data may not be independent given the model, and interval estimates may be too narrow. The reason is that the assumption that the data are independent given the model implies that each data point is informative only about the parameters of the model, not about the other data points. If this is a nonlinear model, then point estimates of the overly constrained parameters may be asymptotically biased, because even when the model is fitted to non-aggregated data, such estimates are effectively based on averaged or summed data, and both kinds of transformations are linear.

Let $\epsilon$ denote the distance from a point estimate to its true value. It seems that at least two conditions have to be met to assess bias magnitude in terms of the validity of substantive conclusions. One is that the model has to be causal, which in this case, it already is. The other is that $\epsilon$ has to be assessed relative to the decision criterion used when arriving at the substantive conclusion, such as the associated interval estimate. For instance, even if $\epsilon$ seems relatively small in absolute terms, but the target value is outside the $95 \%$ interval estimate, and the substantive conclusion (i.e., the causal or the psychological interpretation of the estimate) is based on this interval, then the substantive conclusion will be false. This is not yet a reason for concern since this may merely be the result of sampling error, and sampling error is unavoidable. However, if the true values tend to fall outside the $95 \%$ interval estimates, then the conclusions based on such intervals will tend to be false, and so they will be invalid.

The point and interval estimates obtained by fitting the true and the simplified model are compared with the true values in Fig. 7 below. 


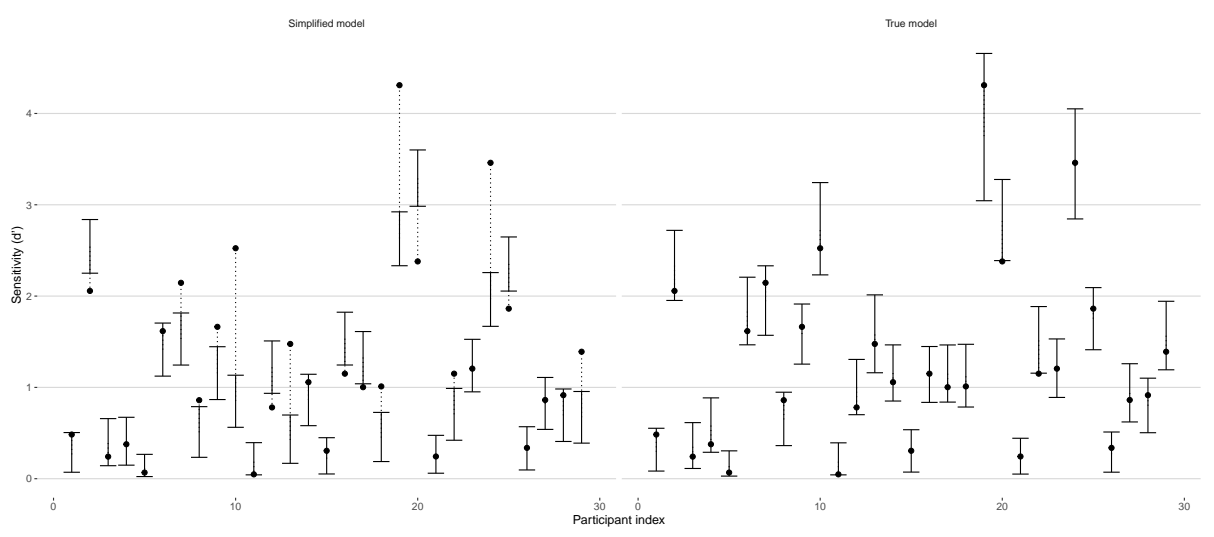

Fig. 7: Point and interval estimate bias caused by ignoring individual differences in the pattern of thresholds' placement. The points represent true individual sensitivities; the intervals represent $95 \%$ credible intervals based on the overly constrained model (left) and the true model (right). The data were simulated from the true model. The dotted lines that mark the distance from the true values to the corresponding interval estimates were introduced to improve readability.

On average, the point estimates differed from the true values by 0.89 and 3.24 standard deviations of the posterior distributions in the case of the true and the simplified model respectively. As much as 55 percent of the true values were outside the $95 \%$ credible intervals based on the simplified model, whereas 7 percent (two instances) of the true values were outside the $95 \%$ credible intervals based on the true model. The $95 \%$ credible intervals for the fixed effect of sensitivity from both models contained the single true value, but the standard deviation of the posterior distribution of the fixed effect of sensitivity from the simplified model was shorter than that from the true model by 23 percent. On average, the standard deviations of the posterior distributions of the thresholds' fixed effects from the simplified model were shorter than the correct intervals by 84 percent. All the $95 \%$ credible intervals for the thresholds' fixed effects from the true model contained the true values, but three out of seven (43 percent) such intervals from the simplified model did not. As can be seen, using the simplified model resulted in severe point and interval estimate bias, and so any substantive conclusions based on this model and this criterion would be anything but valid.

As we have said earlier, by design, the threshold parameters in the simplified model do not correspond to any meaningful property of the data generating process as represented by the causal SDT model. The results clearly indicate that this is also true of the person-specific sensitivity parameters. This illustrates how the interdependence of the estimates of different parameters can lead to the "spread" of bias.

Thanks to the existence of the Confidence Database (Rahnev et al., 2020), we were able to perform the same kind of analysis on many other similar datasets. We cannot summarize the results of those analyses without writing a dedicated paper, but we encourage the skeptical readers to try and do it by adapting our code. The results indicate that typically, both fixed and random effects' estimates tend to be 
associated with severe point and interval estimate bias when the thresholds are assumed to be the same for every participant.

\section{What both analyses illustrate}

In psychology, at least some causal assumptions and some statistical assumptions of the model (e.g., that the process is stationary, that learning does not take place, that the residuals have a normal distribution with constant variance, or that all the effects are linear) are usually known to be false a priori. From this it follows that neither frequentist tests nor any statistical model comparison methods, such as the likelihood ratio test, the information criteria, or the Bayes factor, can be interpreted as evidence that the model is true or false, or that certain values of the target causal quantities can be excluded with some known probability of error. The statistical model comparison methods and model fit indices in particular can only be indicative of the relative predictive performance of the models. By itself, predictive performance does not say anything about the validity of substantive interpretations of estimates of model parameters. In fact, it is easy to come up with realistic examples of pairs of true statistical models such that the model that gives asymptotically biased estimates of the causal target is strongly preferred, by any statistical model comparison method, to the model that gives the correct estimates. That is why we did not use such methods, and we interpreted the interval estimates strictly as measures of uncertainty that are relative to a false model.

Although so far literature on this seems to be lacking, in our experience, when latent psychological attributes, processes, or states are the subject of study, the typical result of attempts to use causal diagrams as aids in understanding the meaning of the obtained results of statistical analysis is a quick realization of a hard limit on what can in principle be inferred from available data. An honest way out of this situation seems to be to try and identify theoretically possible reasons why the main conclusions could be wrong and maybe perform some kind of causal sensitivity analysis.

In that spirit, equipped with the causal definition of measurement invariance, we can say that both analyses aimed at assessing the extent of causal measurement bias. The purpose of the first analysis was to evaluate the model by assessing the conditional independencies implied by the causal assumptions, while the purpose of the second analysis was to assess the possible magnitude of causal estimate bias by simulating hypothetical sources of bias. This kind of theoretically-guided causal model search and focused model testing is recommended as a way of evaluating and improving substantive models by Pearl et al. (2016) and McElreath (2020), among others.

The starting point of the first analysis was the introduction of the qualitative causal definitions of all the relevant quantities and relations. This led to the conclusion that, given the design and the current state of knowledge, all the estimates of model parameters were asymptotically biased as estimates of the target causal quantities.

Despite the fact that the model was clearly false and the estimates of all of its parameters were biased as estimates of the causal targets, there was no evidence that the threshold parameters were influenced by the stimulus class. This supports 
the idea that the estimates of threshold parameters, although biased, may perhaps be useful as estimates of actual decision thresholds, that the estimates of sensitivity parameters, although they are biased as well, may perhaps be useful as estimates of actual sensitivity, and that the identified distributions of latent samples may be useful as approximations of the distributions of internal evidence samples on which the participants' decisions were based.

The first analysis can be generalized in various ways. For instance, the selectivity of influence of the stimulus class in a UV SDT model corresponds to two kinds of effects in the more general cumulative model: the thresholds can be uniformly shifted, and the distances between adjacent thresholds can increase or decrease in a uniform fashion. By using a contrast just like the one described earlier, except that in this new contrast, the thresholds' positions are divided by the average inter-threshold distance, we could test if the stimulus class influences the thresholds according to the UV SDT model. Or, we could relax the normality assumption and estimate the similarity of the pattern of the two sets of thresholds when the normal distribution is replaced with some other distribution.

In fact, by showing which set of assumptions results in the strongest apparent selectivity of the influence of the stimulus class, we could perhaps obtain evidence that the estimates of sensitivity and threshold parameters of the corresponding model best represent actual sensitivity and decision thresholds. This idea is closely related to the causal but not causal-theoretic method of looking for separate modifiability (Sternberg, 2001), which was applied, with mixed results, to SDT models (see, e.g., Sternberg, 2001; Alsop, 1998). However, instead of looking for factors that each selectively influence different measures, we propose to look for the model that results in the strongest apparent selectivity of the effect of the factor that by definition is supposed to selectively influence a particular measure.

The first analysis also illustrates that putting the theory before the method may require that not only is the interpretation of the results rather modest, but also that the theoretical assumptions are weak; in particular, theory-driven causal model search seemed to have some merit in this case partly because of how weak the definition of sensitivity in the causal version of SDT was: it was just the causal effect of the stimulus class on the latent mean.

As the second analysis illustrates, a lot can go wrong when ordinal data are analysed with ordinal models. The results of this analysis should not come as a surprise for at least three reasons. An obvious one is that almost any model can be constrained to the point that it can only be used for prediction. By assuming that the thresholds do not differ between individuals, the SDT model was stripped of the one property that allows, if the model is true, for the separation of sensitivity and threshold effects. Another, more general reason is that not accounting for the variability in a parameter of an arbitrary nonlinear model may lead to asymptotic point and interval bias of the estimate of that parameter and of all the parameters the estimates of which depend on the overly constrained parameter. The final reason, and this is where we allow ourselves to speculate, is that, unlike the normal distribution, the multinomial distribution does not have a separate variance parameter, and so also for that reason, the neglected variability in the thresholds may have had some impact on the point or interval estimates of the sensitivity parameters. Perhaps somewhat paradoxically then, the fact that the SDT model with item parameters was quite appropriately a nonlinear model and that it quite 
appropriately represented the distribution of discrete response as a multinomial distribution may have made it more susceptible to point or interval estimate bias.

It may seem that the problems that we focused on in the first or in the second analysis can be addressed by testing if the candidate estimates of latent variables correlate with theoretically related criteria. This is probably the most common approach to test validation even though it has been known for some time not to work (Borsboom, Romeijn, \& Wicherts, 2008; Millsap, 1997, 2007). We can illustrate this point as well; in the second analysis, the correlation between the severely biased sensitivity estimates and the best criterion there is, i.e., the known true values, was $r=.77$. This shows that the predictive, or, in general, the practical usefulness of a measure, is very weakly related to its unbiasedness. More generally, to assess the extent of measurement bias, one has to show if certain offending causal relations are accounted for or if they are negligible. Even in principle, this cannot be achieved by showing that certain correlations can be reliably detected. That is because measurement invariance cannot be established by any statistical method.

It is far from obvious if the problem observed in the second analysis is less or more severe in situations where IRT models are typically used. One would hope that the variability of the total score is mostly due to the target latent variable, i.e., that reliability is high, and so the kind of bias that we have just illustrated may be less of an issue. However, in this analysis, the correlation between the total score, i.e., the sum of correct responses, and the known true latent values, i.e., the sensitivity, was $r=.9$. Add to this the fact that the typical effect size in some psychological subdisciplines is rather small (see, e.g., Schäfer \& Schwarz, 2019), and when it is small, the observed patterns of response can in principle be in part or in their entirety attributed to some hard to identify factors that contribute only a small part of the variance. Finally, while there is reason to believe that an SDT model is perhaps not entirely inappropriate as a model of the response process, at least in the case of simple perceptual, attentional, or memory tasks, for the reasons that we have already provided, the same cannot be said about any IRT model as a model of the process of responding to the items on self-report tests of state, not to mention tests of latent constructs that are causally further from the response, such as personality traits or intelligence.

Even though we can safely assume that none of the models that were used in both analyses was true, we can also say that an SDT model with person-specific thresholds was more realistic than the model without person-specific thresholds in an important, partially psychological sense. To assess the impact of one unrealistic simplification, we used certain measures of interval and point estimate bias. Since the measures were used only after realizing one of the false models by simulation, we cannot say much about the actual bias resulting from fitting the simplified model to the real data. Consequently, all we can say is that the observed point and interval estimate bias may be illustrative of the magnitude of bias that may occur when the simplified model is used. If anything, the problem is probably more severe than the simulation indicates.

This mirrors an important aspect of causal inference. Unless the true quantitative causal model (i.e., the structural model, see Pearl et al., 2016) is known, and in psychology it never is, causal inference is first and foremost a theoretical exercise in speculative reasoning: When the true model is not known, an arrow in the assumed causal graph usually represents the mere possibility of influence of an 
arbitrary kind. Therefore, it is the lack of an arrow, such as the lack of an arrow from individuals to item parameters, that represents an actual assumption in such models.

Even though we explicitly assumed that neither model is true, and so we cannot say that the results provide any evidence that one of the models may be true, by performing a focused causal test and a simple causal sensitivity analysis, we obtained some evidence that one of the models seems to be more useful for certain purposes. This kind of reasoning only requires that the models can be compared in terms of psychological realism, not that one of the models is true.

We should stress that there is really nothing new about the idea of causal sensitivity analysis: fake-data simulation is often used in Bayesian data analysis to ensure that the model performs as expected, and, more importantly in this context, it is recognized as sometimes the only way to test if a model can reliably recover latent parameter values (Gelman et al., 2020). This is also how the impact of individual differences in the way of responding on test performance is sometimes studied (see, e.g., Asûn et al., 2017).

The approach exemplified by the two analyses can be extended in various ways: for example, by performing statistical sensitivity analysis (how do the priors affect the conclusions?) or by testing other kinds of models (are the results similar if we relax the assumption of equal variance? what about non-normal distributions?). Importantly, even though the number of measurements per participant per item may sometimes be too small for a more realistic model to be identifiable, this is not required by the kind of speculative reasoning that we advocate for.

Simulational investigation of possible bias sidesteps the essential difficulty of item bias detection methods, at a price. As noted by Millsap, bias detection methods rely on assumptions that are hard if not impossible to verify in practice. The heart of the matter is that item bias is defined in terms of distributions of the response of individuals who are matched on the target dimension. It follows that to detect bias, one has to solve a sometimes-impossible task: In observational studies, to ensure that individuals or groups are matched on the target dimension, one has to measure the target dimension, but the reason one tries to measure it is that the measure is assumed to be problematic in hard-to-predict ways.

Therefore, we suggest that this may indeed be an impossible task as long as the target is not a causal property that can be estimated given the theoretically justified causal assumptions and study design. In less favourable conditions, bias detection methods should then perhaps be entirely replaced with a fundamentally different approach. Instead of pretending or hoping that certain very strong assumptions are true, the researcher can weaken the assumptions by considering more complex and more realistic causal models. Granted, this is not a way to detect bias, but it is a perfectly legitimate way of taking it into account.

All that is necessary to speculate about causal estimate bias in this way is that at least one alternative realistic causal model is considered. It is of course preferable that this competing model was fitted to the data, but nothing in the line of reasoning presented so far requires that it is. For instance, the values of the parameters of a hypothetical measurement model without item parameters can be based on an already fitted model that has item parameters. Variability in the item parameters can then be artificially introduced by sampling from a theoretically justified distribution. 
The competing model in which the artificially added complexity is simulated may be more realistic despite the fact that, since it is artificially created, it is also in a sense "less true". That being said, we emphasize again that this kind of simulation can help speculate about the possible magnitude of estimate bias, but it cannot indicate in what way specifically the results obtained are systematically distorted, i.e., it cannot remove bias. In fact, given the myriad factors, many of them unobservable, that can affect the process of response, and the fact that the statistical part of the model is probably never true, we would not be surprised if removing bias turned out to be impossible to achieve even in experimental studies on relatively simple processes.

\section{Concluding remarks}

In the case of an experiment, unless the researcher is interested only in the observed patterns of response to external stimuli or conditions, alternative causal explanations in terms of latent variables and unobservable processes have to be considered. When a latent variable is measured without randomisation, the result of measurement is never an end in itself; it is obtained to predict something else, to study the method itself, or to estimate and understand the pattern of statistical dependencies between the response and some other variables. In every case, alternative explanations of the already known or the just-observed statistical relations play an essential role. Central to the approach to measurement that we tried to follow in this paper is the realization that in psychology, error in measurement of latent variables is mostly systematic. The proposed causal definition of measurement bias was intentionally formulated so that it could be used to formally express, often partial and non mutually exclusive, alternative causal explanations of measurements, as well as alternative causal explanations of the observed statistical dependencies in experimental or observational studies.

Once some plausible alternative explanations are identified, a new form of inquiry may be proposed that aims to rule out or find evidence of their plausibility, and this cycle can continue indefinitely or until there are only relatively implausible alternatives left. Some alternative causal explanations will correspond to biasinducing paths and hypothetical nuisance variables. When the response can be thought of as the result of categorisation of a latent variable, the statistical part of our model can be used to test causal hypotheses and estimate approximations of target causal quantities by exploiting the power of repeated measurement and randomisation, but it can also be useful when the design of the study is weaker, e.g., by providing a framework for causal sensitivity analysis.

\section{Overview of the order-preserving link functions}

We have introduced the first order-preserving link function, now called "softmax", as part of the general hierarchical SDT model that was implemented as an open-source R package called bhsdtr (Paulewicz \& Blaut, 2020). Soon after, the bhsdtr package was replaced with the new version called bhsdtr2 https://github.com/boryspaulewicz/bhsdtr2. In bhsdtr2, a wider range of models was implemented, and new order-preserving link functions were introduced, which are all members of the order-preserving log link function family described here. Note also that there are plans to implement the order-preserving link functions in future versions of the excellent brms package (Bürkner, 2019, 2017). 
What we call an order-preserving link function is a multivariate function that isomorphically maps the space of strictly ordered real vectors, i.e., vectors $\boldsymbol{v}$ such that $\boldsymbol{v}_{i}<\boldsymbol{v}_{j}$ if $i<j$, to the space of unrestricted real vectors $\gamma$ of the same dimension in such a way that the order is preserved. The effects in $\gamma$ can then be modelled with multivariate hierarchical or non-hierarchical linear regression. Typically, every effect will be $K-1$ dimensional, where $K$ is the number of response categories, because every effect in the thresholds can be different for different thresholds. For instance, under dummy contrast coding, the effect of a factor with two levels will be represented by a $K-1$ dimensional intercept (the values of $\gamma$ in the base-level condition) and a $K-1$ dimensional difference between the conditions. Likewise, if random effects in the thresholds are modelled, they will be represented by an at least $K-1$ dimensional multivariate normal distribution (see Paulewicz \& Blaut, 2020, for a detailed explanation of how correlated random effects are handled in bhsdtr and bhsdtr2).

It is important to remember that because every order-preserving link function is nonlinear, the effects in $\gamma$ have to be translated to condition-specific estimates of $\gamma$ before converting them to effects in the thresholds' space. In bhsdtr2, this is handled automatically for every parameter of every model by the function called "samples" (see the package documentation for details).

The new order-preserving link functions are all based on the same simple idea: if we arbitrarily select a single threshold index, the corresponding threshold can be represented directly by an unconstrained parameter, because a decision threshold considered in isolation is an unconstrained parameter. However, when there is more than one threshold, the thresholds above (below) the selected threshold have to remain greater (lower) than the selected threshold, and the same goes for every other pair of adjacent thresholds. Instead of modelling the remaining thresholds separately, it is possible to represent each one as a distance from some adjacent threshold. Assuming that the thresholds cannot collapse, the distances have to be positive, which means that they can be mapped to $\gamma$ using the log function.

This general idea can be realized in many different ways, some of which are perhaps more intuitive than others. For example, if the vector of thresholds $\tau$ has only three elements, we can represent $\tau_{2}$, as the unconstrained parameter $\gamma_{2}, \tau_{1}$ can be represented as $\gamma_{1}=\log \left(\tau_{2}-\tau_{1}\right)$, and $\tau_{3}$ as $\gamma_{3}=\log \left(\boldsymbol{\tau}_{3}-\boldsymbol{\tau}_{2}\right)$. In bhsdtr2, this kind of link function is called "log_distance," and it is used by default in the current version of the package. We have recently learned from the Stan documentation that this kind of function was used internally by Stan for many years as a way to represent ordered thresholds; we are also aware of one other author who just recently independently realized that this kind of function can be used to model ordinal data (Merkle, Fitzsimmons, Uanhoro, \& Goodrich, 2021; Merkle \& Rosseel, 2018). The reader may appreciate that it is often relatively easy to reason about the priors on the $\gamma$ vector under this link function.

The link function family called "log_ratio" is slightly more complicated. The $K / 2$ th element of $\gamma$ directly represents the middle threshold. The $\gamma_{K / 2+1}$ parameter represents $\log \left(\boldsymbol{\tau}_{K / 2+1}-\right.$ $\boldsymbol{\tau}_{K / 2}$ ), which can be thought of as the "spread" parameter, because all the other distances are represented in terms of this one. The $\gamma_{K / 2-1}$ parameter represents the asymmetry between the lower and the upper spread of the thresholds which are adjacent to the main threshold, i.e., the following $\log$-ratio of distances: $\log \left(\left(\boldsymbol{\tau}_{K / 2}-\boldsymbol{\tau}_{K / 2-1}\right) /\left(\boldsymbol{\tau}_{K / 2+1}-\boldsymbol{\tau}_{K / 2}\right)\right)$. The elements $\gamma_{K / 2+i}$, where $i>1$, also represent ratios of distances, i.e., $\gamma_{K / 2+i}=\log \left(\left(\boldsymbol{\tau}_{K / 2+i}-\right.\right.$ $\left.\left.\boldsymbol{\tau}_{K / 2+i-1}\right) /\left(\boldsymbol{\tau}_{K / 2+1}-\boldsymbol{\tau}_{K / 2}\right)\right)$, and we like to call them "upper consistency" parameters. The elements $\gamma_{K / 2-i}$, where $i>1$, are defined as $\gamma_{K / 2-i}=\log \left(\left(\boldsymbol{\tau}_{K / 2-i+1}-\boldsymbol{\tau}_{K / 2-i}\right) /\left(\boldsymbol{\tau}_{K / 2}-\right.\right.$ $\left.\tau_{K / 2-1}\right)$ ), and we like to call them "lower consistency" parameters.

As the two link function families illustrate, ordered thresholds can be mapped isomorphically to the unconstrained space in many different ways using logs of distances and logs of ratios of distances. All such functions are instances of what we call the order-preserving $\log$ link function family. Every element of the set of order-preserving log link functions for $K$ ordered response categories can be obtained using the following algorithm, which we describe only for the $K=4$ case because it is easy to generalize it to arbitrary $K$ :

1. Choose an index $i$ between 1 and $K-1$ - this will be the unconstrained parameter.

2. Represent $\tau_{i}$ directly as $\gamma_{i}$.

3. Choose an index $j$ of a threshold adjacent to $i$.

4. Represent $\tau_{j}$ as $\log$ of distance, i.e., $\gamma_{j}=\log \left(\left|\tau_{j}-\tau_{i}\right|\right)$.

5. If $i \neq 2$ then represent the remaining threshold $\tau_{k}$ as $\log$ of distance between $\tau_{k}$ and $\tau_{j}$ or as $\log$ of ratio of two distances, i.e., $\log \left(\left|\boldsymbol{\tau}_{k}-\boldsymbol{\tau}_{j}\right| /\left|\boldsymbol{\tau}_{j}-\boldsymbol{\tau}_{\boldsymbol{i}}\right|\right)$. If $i=2$ then represent the remaining threshold $\boldsymbol{\tau}_{k}$ as $\log \left(\left|\boldsymbol{\tau}_{k}-\boldsymbol{\tau}_{i}\right|\right)$ or as $\log \left(\left|\boldsymbol{\tau}_{k}-\boldsymbol{\tau}_{i}\right| /\left|\boldsymbol{\tau}_{j}-\boldsymbol{\tau}_{i}\right|\right)$. 
It may seem that one order-preserving link function is enough for general use, but it is not. If there is a numerical predictor in the model, its effect may be approximately linear under some link functions but not others, and so the choice of a link function may determine the number of parameters. The choice of a link function is also important when there are only nominal predictors because it is an open question if random effects in the $\gamma$ space can be approximated well by a multivariate normal distribution. Moreover, the thresholds' fixed effects are defined as population averages of $\gamma$ random effects, just like a fixed effect in hierarchical logistic regression is defined as the population average of group-specific effects on the logit scale; because of nonlinearity, the threshold fixed effects are in general not equal to populationaverage effects on the latent scale. This means that, for example, an effect of a factor may seem different under one link function than under another. The researcher may also suspect a priori that e.g., certain distances may vary with certain predictors, but the ratios of certain distances may stay relatively constant. Such constraints can be directly enforced by choosing an appropriate link function and fixing some of the parameters. Finally, since the link functions are nonlinear, a non-zero effect which is additive in the $\gamma$ space under one link function (or in the $\tau$ space) will be interactive under another link function (or in the $\gamma$ space). In such cases, the apparent interaction will be an artifact of the scale; a failure to recognize this may lead to invalid conclusions.

The GitHub package repository (https://github.com/boryspaulewicz/bhsdtr2) contains the annotated source code and data that were used to perform all the analyses and produce all the figures presented in this paper.

\section{References}

Ackerman, T. A., \& Evans, J. A. (1994). The influence of conditioning scores in performing DIF analyses. Applied Psychological Measurement, 18(4), 329342.

Alsop, B. (1998). Receiver operating characteristics from nonhuman animals: Some implications and directions for research with humans. Psychonomic Bulletin \& Review, 5(2), 239-252.

Anderson, J. R. (2013). The adaptive character of thought. Psychology Press.

Angoff, W. H. (1993). Perspectives on differential item functioning methodology. In P. W. Holland \& H. Wainer (Eds.), (pp. 3-23). Lawrence Erlbaum Associates, Inc.

Asûn, R. A., Rdz-Navarro, K., \& Alvarado, J. M. (2017). The sirens' call in psychometrics: The invariance of IRT models. Theory \& Psychology, 27(3), 389-406.

Borsboom, D. (2005). Measuring the mind: Conceptual issues in contemporary psychometrics. Cambridge University Press.

Borsboom, D., Mellenbergh, G. J., \& Van Heerden, J. (2003). The theoretical status of latent variables. Psychological review, 110(2), 203.

Borsboom, D., Mellenbergh, G. J., \& van Heerden, J. (2004). The concept of validity. Psychological Review, 111(4), 1061.

Borsboom, D., Romeijn, J.-W., \& Wicherts, J. M. (2008). Measurement invariance versus selection invariance: Is fair selection possible? Psychological Methods, $13(2), 75$.

Bürkner, P.-C. (2017). brms: An R package for Bayesian multilevel models using Stan. Journal of Statistical Software, 80(1), 1-28.

Bürkner, P.-C. (2019). Bayesian item response modeling in R with brms and Stan. arXiv preprint arXiv:1905.09501. 
Camilli, G. (1992). A conceptual analysis of differential item functioning in terms of a multidimensional item response model. Applied Psychological Measurement, 16(2), 129-147.

Carpenter, B., Gelman, A., Hoffman, M., Lee, D., Goodrich, B., Betancourt, M., ... Riddell, A. (2016). Stan: A probabilistic programming language. Journal of Statistical Software, 20, 1-37.

Chater, N., \& Oaksford, M. (1999). Ten years of the rational analysis of cognition. Trends in cognitive sciences, 3(2), 57-65.

De Boeck, P. (2004). Explanatory item response models: A generalized linear and nonlinear approach. Springer Science \& Business Media.

Drasgow, F., Levine, M. V., \& Williams, E. A. (1985). Appropriateness measurement with polychotomous item response models and standardized indices. British Journal of Mathematical and Statistical Psychology, 38(1), 67-86.

Franco, V. R., Wiberg, M., \& Laros, J. A. (2021). Situational optimization function analysis: An ideal performance analysis inspired on lewin's equation. Psychological methods.

Gelman, A., Vehtari, A., Simpson, D., Margossian, C. C., Carpenter, B., Yao, Y., ... Modrák, M. (2020). Bayesian workflow. arXiv preprint arXiv:2011.01808.

Green, D. M., \& Swets, J. A. (1966). Signal Detection Theory and Psychophysics. New York: Wiley.

Holland, P. W. (1990). On the sampling theory roundations of item response theory models. Psychometrika, 55(4), 577-601.

Holland, P. W., \& Wainer, H. (2012). Differential item functioning. Routledge.

Junker, B. W., \& Ellis, J. L. (1997). A characterization of monotone unidimensional latent variable models. The Annals of Statistics, 25(3), 1327-1343.

Klein, D. F., \& Cleary, T. A. (1967). Platonic true scores and error in psychiatric rating scales. Psychological Bulletin, 68(2), 77.

Liddell, T. M., \& Kruschke, J. K. (2018). Analyzing ordinal data with metric models: What could possibly go wrong? Journal of Experimental Social Psychology, 79, 328-348.

Lord, F. M., \& Novick, M. R. (1968). Statistical theories of mental test scores. IAP.

Lumsden, J. (1976). Test theory. Annual review of psychology, 27(1), 251-280.

Macmillan, N. A., \& Creelman, C. D. (2004). Detection theory: A user's guide. Psychology Press.

McElreath, R. (2020). Statistical rethinking: A bayesian course with examples in $R$ and Stan. Chapman and Hall/CRC.

Meijer, R. R., \& Sijtsma, K. (2001). Methodology review: Evaluating person fit. Applied Psychological Measurement, 25(2), 107-135.

Mellenbergh, G. J. (1989). Item bias and item response theory. International journal of educational research, 13(2), 127-143.

Mellenbergh, G. J. (1995). Conceptual notes on models for discrete polytomous item responses. Applied Psychological Measurement, 19(1), 91-100.

Merkle, E. C., Fitzsimmons, E., Uanhoro, J., \& Goodrich, B. (2021). Efficient Bayesian structural equation modeling in Stan. Journal of Statistical Software, $100(6), 1-22$.

Merkle, E. C., \& Rosseel, Y. (2018). blavaan: Bayesian structural equation models via parameter expansion. Journal of Statistical Software, 85(4), 1-30. 
Millsap, R. E. (1997). Invariance in measurement and prediction: Their relationship in the single-factor case. Psychological methods, 2(3), 248.

Millsap, R. E. (2007). Invariance in measurement and prediction revisited. Psychometrika, 72(4), 461-473.

Millsap, R. E. (2012). Statistical approaches to measurement invariance. Routledge.

Oaksford, M., \& Chater, N. (Eds.). (1998). Rational models of cognition. Oxford University Press.

Osterlind, S. J., \& Everson, H. T. (2009). Differential item functioning. Sage Publications.

Paulewicz, B., \& Blaut, A. (2020). The bhsdtr package: a general-purpose method of Bayesian inference for Signal Detection Theory models. Behavior Research Methods, 1-20.

Pearl, J. (2000). Causality: Models, reasoning and inference. Cambridge University Press.

Pearl, J., Glymour, M., \& Jewell, N. P. (2016). Causal inference in statistics: A primer. John Wiley \& Sons.

Peterson, W., Birdsall, T., \& Fox, W. (1954). The theory of signal detectability. Transactions of the IRE professional group on information theory, 4(4), 171212.

R Core Team. (2017). R: A language and environment for statistical computing [Computer software manual]. Vienna, Austria. Retrieved from https://www.R-project.org/

Rahnev, D., Desender, K., Lee, A. L., Adler, W. T., Aguilar-Lleyda, D., Akdoğan, B., ... others (2020). The confidence database. Nature human behaviour, $4(3), 317-325$.

Ramsøy, T. Z., \& Overgaard, M. (2004). Introspection and subliminal perception. Phenomenology and the Cognitive Sciences, 3(1), 1-23.

Rasch, G. (1960). Studies in mathematical psychology: I. probabilistic models for some intelligence and attainment tests. Nielsen \& Lydiche.

Ratcliff, R. (1978). A theory of memory retrieval. Psychological review, 85(2), 59.

Ratcliff, R., \& McKoon, G. (2008). The diffusion decision model: Theory and data for two-choice decision tasks. Neural computation, 20(4), 873-922.

Samejima, F. (1968). Estimation of latent ability using a response pattern of graded scores 1. ETS Research Bulletin Series, $1968(1)$, i-169.

Schäfer, T., \& Schwarz, M. A. (2019). The meaningfulness of effect sizes in psychological research: Differences between sub-disciplines and the impact of potential biases. Frontiers in psychology, 10, 813.

Spielberger, C. D., Gorusch, R. L., Lushene, P. R., Vagg, P. R., \& Jacobs, A. G. (1983). Manual for the state-trait anxiety inventory (form y). Consulting Psychologists Press, Inc.

Sternberg, S. (2001). Separate modifiability, mental modules, and the use of pure and composite measures to reveal them. Acta Psychologica, 106(1), $147-246$.

Tanner Jr, W. P., \& Swets, J. A. (1954). A decision-making theory of visual detection. Psychological Review, 61(6), 401.

Tuerlinckx, F., \& Boeck, P. D. (2005). Two interpretations of the discrimination parameter. Psychometrika, 70(4), 629-650. 
van Bork, R., Rhemtulla, M., Sijtsma, K., \& Borsboom, D. (2020). A causal theory of error scores. PsyArXiv.

Van Der Ark, L. A. (2001). Relationships and properties of polytomous item response theory models. Applied Psychological Measurement, 25(3), 273282.

Van der Linden, W. J. (2018). Handbook of item response theory: Three volume set. CRC Press.

van der Maas, H. L., Molenaar, D., Maris, G., Kievit, R. A., \& Borsboom, D. (2011). Cognitive psychology meets psychometric theory: on the relation between process models for decision making and latent variable models for individual differences. Psychological review, $118(2), 339$.

Wickens, T. D. (2002). Elementary Signal Detection theory. Oxford University Press, USA.

Zumbo, B. D. (2007). Three generations of DIF analyses: Considering where it has been, where it is now, and where it is going. Language assessment quarterly, $4(2), 223-233$. 